\title{
A bargaining procedure leading to the serial rule in games with veto players
}

\author{
J. Arin, V. Feltkamp ${ }^{\dagger}$ and M. Monter ${ }^{\ddagger}$ \\ Final version accepted by Annals of Operations Research, March 2015 \\ The final publication is available at Springer via \\ http://dx.doi.org/10.1007/s10479-015-1841-5
}

\begin{abstract}
This paper studies an allocation procedure for coalitional games with veto players. The procedure is similar to the one presented by Arin and Feltkamp (J Math Econ 43:855-870, 2007), which is based on Dagan et al. (Games Econ Behav 18:55-72, 1997). A distinguished player makes a proposal that the remaining players must accept or reject, and conflict is solved bilaterally between the rejector and the proposer. We allow the proposer to make sequential proposals over several periods. If responders are myopic maximizers (i.e. consider each period in isolation), the only equilibrium outcome is the serial rule of Arin and Feltkamp (Eur J Oper Res 216:208-213, 2012) regardless of the order of moves. If all players are fully rational, the serial rule

*Dpto. Ftos. A. Económico I, University of the Basque Country, L. Agirre 83, 48015 Bilbao, Spain. Email: franciscojavier.arin@ehu.es.

$\dagger$ Maastricht School of Management, PO Box 1203, 6201 BE Maastricht, The Netherlands. Email: Feltkamp@msm.nl.

${ }^{\ddagger}$ School of Economics, University of Nottingham, Nottingham NG7 2RD, UK. E-mail: maria.montero@nottingham.ac.uk.

§IKERBASQUE, Basque Foundation of Science and Dpto. Ftos. A. Económico I, University of the Basque Country, Bilbao, Spain.
\end{abstract}


still arises as the unique subgame perfect equilibrium outcome if the order of moves is such that stronger players respond to the proposal after weaker ones.

Keywords: game theory, veto players, bargaining, serial rule.

JEL classification: C71, C72, C78, D70.

\section{Introduction}

Consider a multilateral bargaining situation with one distinguished player (the most senior creditor in the bankruptcy case, the chair of a committee, the manager of a firm...). The distinguished player negotiates bilaterally with each of the other players. Negotiations are constrained by a fairness or justice principle that is commonly accepted in society and can be enforced (possibly by an external court). Players are assumed to be selfish, hence they only appeal to this principle when it is in their material interest to do so. To what extent does the global agreement reflect the bilateral principle? In Dagan et al. (1997) the answer is that the bilateral principle completely determines the outcome: if a particular bankruptcy rule can be enforced in the bilateral comparison between the proposer and each responder, the outcome is the same bankruptcy rule applied to the case of $n$ creditors. ${ }^{1}$

Dagan et al.'s paper focuses on bankruptcy games, hence their justice principles are also restricted to this class. The question arises of what the appropriate justice principle should be for general TU games. In this paper we use the (restricted) standard solution of a reduced game between the two players. The idea behind this principle is that each of the two players gains (or loses) the same amount with respect to an alternative situation in which the two players cannot cooperate with each other (unless this would result in a negative payoff for one of the players, in which case this player gets zero).

Using this bilateral principle, Arin and Feltkamp (2007) studied the bargaining procedure in another class of games with a distinguished player,

\footnotetext{
${ }^{1}$ The procedure in Dagan et al. (1997) is based on an earlier paper by Serrano (1995). A variant of this procedure was later studied by Chang and $\mathrm{Hu}$ (2008).
} 
namely games with a veto player. A veto player is a player whose cooperation is essential in order for a coalition to generate value. Games with a veto player arise naturally in economic applications. Examples include a production economy with one landowner and many landless peasants (Shapley and Shubik (1967)), an innovator trading information about a technological innovation with several producers (Muto (1986), Muto et al. (1989), Driessen et al. (1992)) and hierarchical situations where a top player's permission is necessary in order for a project to be developed (Gilles et al. 1992). Arin and Feltkamp (2007) found that the equilibria of this bargaining procedure are not always efficient: the proposer may be strictly better-off by proposing an allocation that does not exhaust the total available payoff.

In the present paper, we modify the above procedure by allowing the proposer to make a fixed number of sequential proposals, so that players can continue bargaining over the remainder if the first proposal did not exhaust the value of the grand coalition. Each period results in a partial agreement, and then a new TU game is constructed where the values of the coalitions take into account the agreements reached so far; the final outcome is the sum of all partial agreements. We assume that the number of available bargaining periods $T$ is at least as large as the number of players $n$. In order to analyze this multiperiod game, we start by a simplified model in which responders behave myopically, that is, we initially assume that responders consider each period in isolation, accepting or rejecting the current proposal without anticipating the effects of their decision on future periods. The proposer is assumed to behave rationally, taking into account the effect of his actions on future periods and also taking into account that the responders behave myopically. We refer to this kind of strategy profile as a myopic best response equilibrium.

It turns out that all myopic best response equilibria are efficient and lead to the same outcome, which is the serial rule of Arin and Feltkamp (2012). This solution concept is based on the idea that the strength of player $i$ can be measured by the maximum amount a coalition can obtain without player $i$, denoted by $d_{i}$. Since it is impossible for any coalition to obtain a payoff above $d_{i}$ without $i$ 's cooperation, player $i$ can be viewed as having a veto 
right over $v(N)-d_{i}$. The serial rule divides $v(N)$ into segments, and each segment is equally divided between the players that have a veto right over it.

We then turn to the analysis of subgame perfect equilibrium outcomes and show that they may differ from the serial rule. The order of moves may be such that the proposer is able to hide some payoff from a stronger player with the cooperation of a weaker player: the proposal faced by the stronger player is not too favorable for the proposer so that the stronger player cannot challenge it, but later on a weak player rejects the proposal and transfers some payoff to the proposer; the weak player may have an incentive to do so because of the effect of this agreement on future periods. However, if the order of moves is such that stronger players have the last word in the sense that they respond to the proposal after weaker ones, the only subgame perfect equilibrium outcome is the serial rule. Hence, myopic and rational behavior of the responders lead to the same outcome in this case.

\section{Preliminaries}

\section{$2.1 \quad$ TU games}

A cooperative n-person game in characteristic function form is a pair $(N, v)$, where $N$ is a finite set of $n$ elements and $v: 2^{N} \rightarrow \mathbb{R}$ is a real-valued function on the family $2^{N}$ of all subsets of $N$ with $v(\emptyset)=0$. Elements of $N$ are called players and the real-valued function $v$ the characteristic function of the game. Any subset $S$ of the player set $N$ is called a coalition. The number of players in a coalition $S$ is denoted by $|S|$. In this work we will only consider games where all coalitions have nonnegative worth and the grand coalition is efficient, that is, $0 \leq v(S) \leq v(N)$ for all $S \subset N$.

A payoff allocation is represented by a vector $x \in \mathbb{R}^{n}$, where $x_{i}$ is the payoff assigned by $x$ to player $i$. We denote $\sum_{i \in S} x_{i}$ by $x(S)$. If $x(N) \leq v(N)$, $x$ is called a feasible allocation; if $x(N)=v(N), x$ is called an efficient allocation. An efficient allocation satisfying $x_{i} \geq v(i)$ for all $i \in N$ is called an imputation and the set of imputations is denoted by $I(N, v)$. The set of 
nonnegative feasible allocations is denoted by $D(N, v)$ and formally defined as follows

$$
D(N, v):=\left\{x \in \mathbb{R}^{N}: x(N) \leq v(N) \text { and } x_{i} \geq 0 \text { for all } i \in N\right\} .
$$

A (single-valued) solution $\phi$ on a class of games $\Gamma$ is a function that associates with every game $(N, v)$ in $\Gamma$ a feasible allocation $\phi(N, v)$ in $\mathbb{R}^{N}$. The solution $\phi$ satisfies the aggregate monotonicity property (Meggido, 1974) on the class of games $\Gamma$ if the following holds: for all $v, w \in \Gamma$ such that $v(S)=w(S)$ for all $S \neq N$ and $v(N)<w(N)$, then $\phi_{i}(N, v) \leq \phi_{i}(N, w)$ for all $i \in N$. Increasing the value of the grand coalition never leads to a payoff decrease for any of the players.

The core of a game is the set of imputations that cannot be blocked by any coalition, i.e.

$$
C(N, v):=\{x \in I(v): x(S) \geq v(S) \text { for all } S \subseteq N\} .
$$

A game with a nonempty core is called a balanced game. A player $i$ is a veto player if $v(S)=0$ for all coalitions where player $i$ is not present. A game $v$ is a veto-rich game if it has at least one veto player and the set of imputations is nonempty. A balanced game with at least one veto player is called a veto balanced game. Note that balancedness is a relatively weak property for games with a veto player, since it only requires $v(N) \geq v(S)$ for all $S \subset N$.

Given a game $(N, v)$ and a feasible allocation $x$, the excess of a coalition $S$ at $x$ is defined as $e(S, x):=v(S)-x(S)$. Its mirror concept, the satisfaction of a coalition $S$ at $x$, is defined as $f(S, x):=x(S)-v(S)$. We define $f_{i j}(x,(N, v))$ as the minimum satisfaction of a coalition that contains $i$ but not $j$.

$$
f_{i j}(x,(N, v)):=\min _{S: i \in S \subseteq N \backslash\{j\}}\{x(S)-v(S)\} .
$$

If there is no confusion we write $f_{i j}(x)$ instead of $f_{i j}(x,(N, v))$. The higher $f_{i j}(x)$, the better $i$ is treated by the allocation $x$ in comparison with $j$. The kernel can be defined as the set of imputations that satisfy the following bilateral kernel conditions:

$$
f_{j i}(x)>f_{i j}(x) \text { implies } x_{j}=v(j) \text { for all } i, j \text { in } N \text {. }
$$


Note that, if $j$ is a veto player, $f_{i j}(x)=x_{i}{ }^{2}$

Arin and Feltkamp (1997) show that the kernel is a single point for vetorich games. In other words, the nucleolus (Schmeidler, 1969) and the kernel coincide.

\subsection{One-period bargaining (Arin and Feltkamp, 2007)}

Given a veto balanced game $(N, v)$ where player 1 is a veto player and an order on the set of the remaining players, we will define an extensive-form game associated to the TU game and denote it by $G(N, v)$. The game has $n$ stages and in each stage only one player takes an action. In the first stage, a veto player announces a proposal $x^{1}$ that belongs to the set of feasible and nonnegative allocations of the game $(N, v)$. In the next stages the responders accept or reject sequentially. If a player, say $i$, accepts the proposal $x^{s-1}$ at stage $s$, he receives the payoff $x_{i}^{s-1}$ and for the next stage the proposal $x^{s}$ coincides with the proposal at $s-1$, that is $x^{s-1}$. If player $i$ rejects the proposal, this rejection is understood as an appeal for the bilateral fairness principle to be enforced. A two-person TU game is constructed by applying the definition of the Davis-Maschler reduced game ${ }^{3}$ on the set $\{1, i\}$ given $x^{s-1}$, and player $i$ receives as payoff the restricted standard solution $n^{4}$ of this

\footnotetext{
${ }^{2}$ An equivalent definition of the kernel is based on the mirror concept of $f_{i j}$,
} which is the surplus of $i$ against $j$ at $x$ (terminology of Maschler, 1992), $s_{i j}(x):=$ $\max _{S: i \in S \subseteq N \backslash\{j\}}\{v(S)-x(S)\}$. The kernel is the set of imputations such that $s_{i j}(x)>s_{j i}(x)$ implies $x_{j}=v(j)$. We found it more convenient to work with $f_{i j}($.$) rather than s_{i j}($.$) .$

${ }^{3}$ Let $(N, v)$ be a game, $T$ a subset of $N$ such that $T \neq N, \emptyset$, and $x$ a feasible allocation. The Davis-Maschler (1965) reduced game on $T$ given $x$ is the game $\left(T, v_{x}^{T}\right)$ where

$$
v_{x}^{T}(S):=\left\{\begin{array}{l}
0 \quad \text { if } S=\emptyset \\
x(T) \text { if } S=T \\
\max _{Q \subseteq N \backslash T}\{v(S \cup Q)-x(Q)\} \quad \text { for all other } S \subset T .
\end{array}\right.
$$

See also Peleg (1986).

${ }^{4}$ The standard solution of a two-person TU game $v$ gives player $i=1,2$ the amount $v(i)+\frac{v(1,2)-v(i)-v(j)}{2}$. The restricted standard solution coincides with the standard solution except when the standard solution gives a negative payoff to one of the players, in which case this player receives 0 and the other player receives $v(1,2)$. 
two-person game, unless this would result in a negative payoff in which case $i$ receives 0 . Once all the responders have played and consequently have received their payoffs the payoff of the proposer is also determined as $x_{1}^{n}$.

Formally, the resulting outcome of playing the game can be described by the following algorithm.

Input : a veto balanced game $(N, v)$ with a veto player, player 1 , and an order on the set of remaining players (responders). Output : a feasible and nonnegative allocation $x^{n}(N, v)$.

1. Start with stage 1. Player 1 makes a feasible and nonnegative proposal $x^{1}$ (not necessarily an imputation). The superscript denotes at which stage the allocation emerges as the proposal in force.

2. In the next stage the first responder (say, player 2) says yes or no to the proposal. If he says yes he receives the payoff $x_{2}^{1}$ and $x^{2}=x^{1}$.

If he says no he receives the payoff ${ }^{5}$

$$
\begin{array}{r}
y_{2}=\max \left\{0, \frac{1}{2}\left[x_{1}^{1}+x_{2}^{1}-v_{x^{1}}^{\{1,2\}}(1)\right]\right\} \text { where } \\
v_{x^{1}}^{\{1,2\}}(1):=\max _{1 \in S \subseteq N \backslash\{2\}}\left\{v(S)-x^{1}(S \backslash\{1\})\right\} \\
\text { Now, } x_{i}^{2}=\left\{\begin{array}{cc}
x_{1}^{1}+x_{2}^{1}-y_{2} & \text { for player } 1 \\
y_{2} & \text { for player } 2 \\
x_{i}^{1} & \text { if } i \neq 1,2 .
\end{array}\right.
\end{array}
$$

3. Let the stage $s$ where responder $k$ plays, given the allocation $x^{s-1}$. If he says yes he receives the payoff $x_{k}^{s-1}$, and $x^{s}=x^{s-1}$.

If he says no he receives the payoff

$$
y_{k}=\max \left\{0, \frac{1}{2}\left[x_{1}^{s-1}+x_{k}^{s-1}-v_{x^{s-1}}^{\{1, k\}}(1)\right]\right\} \text { where }
$$

\footnotetext{
${ }^{5}$ Note that, since 1 is a veto player, $v_{x^{s}}^{\{1, i\}}(i)=0$ for any proposal $x^{s}$ and any player $i \neq 1$.
} 


$$
\begin{gathered}
v_{x^{s-1}}^{\{1, k\}}(1)=\max _{1 \in S \subseteq N \backslash\{k\}}\left\{v(S)-x^{s-1}(S \backslash\{1\})\right\} . \\
\text { Now, } x_{i}^{s}=\left\{\begin{array}{cc}
x_{1}^{s-1}+x_{k}^{s-1}-y_{k} & \text { for player } 1 \\
y_{k} & \text { for player } k . \\
x_{i}^{s-1} & \text { if } i \neq 1, k
\end{array}\right.
\end{gathered}
$$

4. The game ends when stage $n$ is played and we define $x^{n}(N, v)$ as the vector with coordinates $\left(x_{j}^{n}\right)_{j \in N}$.

In this game we assume that the conflict between the proposer and a responder is solved bilaterally. In the event of conflict, the players face a two-person TU game that shows the strength of each player given that the rest of the responders are passive. The responder then receives the restricted standard solution of this game, which is based on the idea that both players should gain or lose equally with respect to the alternative situation in which negotiations break down (subject to the limited liability constraint that player $i$ cannot get a negative payoff).

The $f_{i j}$ values play an important role in this bargaining procedure as the following lemma illustrates.

Lemma 1 (Arin and Feltkamp, (2007)) Suppose player $k$ is facing the proposal $x^{s-1}$ at stage $s$. If $k$ rejects the proposal:

1. Player $k$ receives the payoff $y_{k}=\max \left\{0, \frac{1}{2}\left[f_{k 1}\left(x^{s-1}\right)+f_{1 k}\left(x^{s-1}\right)\right]\right\}$.

2. The proposal $x^{s}$ that emerges from stage $s$ is such that either $f_{k 1}\left(x^{s}\right)=$ $f_{1 k}\left(x^{s}\right)$, or $f_{k 1}\left(x^{s}\right)>f_{1 k}\left(x^{s}\right)$ and $x_{k}^{s}=0$.

Proof. 1. Note that $v_{x^{s-1}}^{\{1, k\}}(1)=\max _{1 \in S \subseteq N \backslash\{k\}}\left\{v(S)-x^{s-1}(S \backslash\{1\})\right\}=$ $-\min _{1 \in S \subseteq N \backslash\{k\}}\left\{x^{s-1}(S \backslash\{1\})-v(S)\right\}=x_{1}^{s-1}-f_{1 k}\left(x^{s-1}\right)$. Recall also that, since player 1 is a veto player, $f_{k 1}\left(x^{s-1}\right)=x_{k}^{s-1}$.

The payoff player $k$ gets after rejecting proposal $x^{s-1}$ is $y_{k}=\max \left\{0, \frac{1}{2}\left[x_{1}^{s-1}+x_{k}^{s-1}-v_{x^{s-1}}^{\{1, k\}}(1)\right]\right\}$. Replacing $v_{x^{s-1}}^{\{1, k\}}(1)$ and $x_{k}^{s-1}$ by their values, we obtain $y_{k}=\max \left\{0, \frac{1}{2}\left[f_{k 1}\left(x^{s-1}\right)+f_{1 k}\left(x^{s-1}\right)\right]\right\}$.

2. The new proposal in place is such that $x_{k}^{s}=y_{k}$ and $x_{1}^{s}=x_{1}^{s-1}+x_{k}^{s-1}-$ $y_{k}$. This leads to new $f_{i j}$ values. For player $k$, since player 1 is a veto player, 
we have $f_{k 1}\left(x^{s}\right)=x_{k}^{s}$. Since the only difference between $x^{s-1}$ and $x^{s}$ is that a (possibly negative) payoff of $x_{k}^{s}-x_{k}^{s-1}$ has been transferred from 1 to $k$, it holds that $f_{1 k}\left(x^{s}\right)=f_{1 k}\left(x^{s-1}\right)-x_{k}^{s}+x_{k}^{s-1}$.

There are two possible cases. If $\frac{1}{2}\left[f_{k 1}\left(x^{s-1}\right)+f_{1 k}\left(x^{s-1}\right)\right] \geq 0$, we have $x_{k}^{s}=y_{k}=\frac{1}{2}\left[f_{k 1}\left(x^{s-1}\right)+f_{1 k}\left(x^{s-1}\right)\right]$. Replacing $x_{k}^{s}$ by $\frac{1}{2}\left[f_{k 1}\left(x^{s-1}\right)+f_{1 k}\left(x^{s-1}\right)\right]$ and $x_{k}^{s-1}$ by $f_{k 1}\left(x^{s-1}\right)$ in the expression for $f_{1 k}\left(x^{s}\right)$ above, we obtain $f_{1 k}\left(x^{s}\right)=$ $f_{1 k}\left(x^{s-1}\right)-\frac{1}{2}\left[f_{k 1}\left(x^{s-1}\right)+f_{1 k}\left(x^{s-1}\right)\right]+f_{k 1}\left(x^{s-1}\right)=\frac{1}{2}\left[f_{k 1}\left(x^{s-1}\right)+f_{1 k}\left(x^{s-1}\right)\right]=$ $x_{k}^{s}=f_{k 1}\left(x^{s}\right)$.

In the second case we have $\frac{1}{2}\left[f_{k 1}\left(x^{s-1}\right)+f_{1 k}\left(x^{s-1}\right)\right]<0$, so that $x_{k}^{s}=$ $y_{k}=0$. Since $f_{k 1}\left(x^{s-1}\right)=x_{k}^{s-1} \geq 0$, this must be due to $f_{1 k}\left(x^{s-1}\right)<0$. The proposal that emerges from stage $s$ has $x_{k}^{s}=f_{k 1}\left(x^{s}\right)=0$. Since $f_{1 k}\left(x^{s}\right)=$ $f_{1 k}\left(x^{s-1}\right)-x_{k}^{s}+x_{k}^{s-1}$ and $x_{k}^{s}>\frac{1}{2}\left[f_{k 1}\left(x^{s-1}\right)+f_{1 k}\left(x^{s-1}\right)\right]$, we have $f_{1 k}\left(x^{s}\right)<$ $f_{1 k}\left(x^{s-1}\right)-\frac{1}{2}\left[f_{k 1}\left(x^{s-1}\right)+f_{1 k}\left(x^{s-1}\right)\right]+f_{k 1}\left(x^{s-1}\right)=\frac{1}{2}\left[f_{k 1}\left(x^{s-1}\right)+f_{1 k}\left(x^{s-1}\right)\right]<$ $0=f_{k 1}\left(x^{s}\right)$.

The set of pure strategies in this game is relatively simple. Player 1's strategy consists of the initial proposal $x^{1}$, which must be feasible and nonnegative. A pure strategy for the responder who moves at stage $s$ is a function that assigns "yes" or "no" to each possible proposal $x^{s-1}$ and each possible history of play. Players are assumed to be selfish, hence player $i$ seeks to maximize $x_{i}^{n}$.

\subsection{Nash equilibrium outcomes of the one-period game}

The set of bilaterally balanced allocations for player $i$ is

$$
F_{i}(N, v):=\left\{x \in D(N, v): f_{j i}(x) \geq f_{i j}(x) \text { for all } j \neq i\right\}
$$

while the set of optimal allocations for player $i$ in the set $F_{i}(N, v)$ is defined as follows:

$$
B_{i}(N, v):=\underset{x \in F_{i}(N, v)}{\arg \max } x_{i}
$$

In the class of veto balanced games, $F_{i}(N, v)$ is a nonempty and compact set for all $i$, thus the set $B_{i}(N, v)$ is nonempty.

Theorem 1 (Arin and Feltkamp, 2007) Let $(N, v)$ be a veto balanced $T U$ game and let $G(N, v)$ be its associated extensive form game. Let $z$ be a 
feasible and nonnegative allocation. Then $z$ is a Nash equilibrium outcome if and only if $z \in B_{1}(N, v)$.

The intuition behind this result is as follows. Recall that, as shown in lemma 1, the restricted standard solution that is applied if player $i$ rejects a proposal in stage $s$ results in $f_{1 i}\left(x^{s}\right)=f_{i 1}\left(x^{s}\right)$, unless this would mean a negative payoff for player $i$, in which case $f_{i 1}\left(x^{s}\right)>f_{1 i}\left(x^{s}\right)$ and $x_{i}^{s}=0$. Hence, rejection of a proposal leads to a payoff redistribution between 1 and $i$ until the bilateral kernel condition is satisfied between the two players. It is in player $i$ 's interest to reject any proposal with $f_{1 i}\left(x^{s-1}\right)>f_{i 1}\left(x^{s-1}\right)$ and to accept all other proposals. Since player $i$ rejects proposals with $f_{1 i}\left(x^{s-1}\right)>f_{i 1}\left(x^{s-1}\right)$ and this rejection results in $f_{1 i}\left(x^{s}\right)=f_{i 1}\left(x^{s}\right)$, the proposal in force after $i$ has the move always satisfies $f_{i 1}\left(x^{s}\right) \geq f_{1 i}\left(x^{s}\right)$. Subsequent actions by players moving after $i$ can only reduce $f_{1 i}($.$) , hence f_{i 1}\left(x^{n}\right) \geq f_{1 i}\left(x^{n}\right)$. Conversely, player 1 can achieve any bilaterally balanced payoff vector by proposing it. Player 1 then maximizes his own payoff under the constraint that the final allocation has to be bilaterally balanced.

The nucleolus is a natural candidate to be an equilibrium outcome since it is the only efficient allocation that satisfies the bilateral kernel conditions in this class of games, and indeed the nucleolus is always in $F_{1}(N, v)$. However, it is not necessarily in $B_{1}(N, v)$. Instead, Arin and Feltkamp (2007) show that the proposer may be better-off by proposing an inefficient allocation, as the following example illustrates:

Example 1 (Arin and Feltkamp, 2007) $N=\{1,2,3,4,5\} ; v(S)=8$ if $1 \in S$ and $|S|=4 ; v(N)=12$.

The nucleolus of this game is $(4,2,2,2,2)$. However, player 1 can do better by proposing the inefficient allocation $(8,0,0,0,0)$. This inefficient allocation would emerge as the final outcome since it satisfies the bilateral principle. Suppose player 1 sets $x^{1}=(8,0,0,0,0)$ and player 2 rejects the proposal. Player 1 can form a coalition with the other three players and pay them 0 , hence $v_{x^{1}}^{\{1,2\}}(1)=8$. Since player 1 is a veto player, $v_{x^{1}}^{\{1,2\}}(2)=0$. The standard solution of the reduced game allocates 0 to player 2 and leaves the proposal unchanged, $x^{2}=(8,0,0,0,0)$. Note that allocation $(8,0,0,0,0)$ is 
the nucleolus of a game $w$ that coincides with $v$ except that $w(N)=x(N)=$ 8. Since the nucleolus does not satisfy aggregate monotonicity for the class of veto balanced games, the proposer may be better-off by proposing the nucleolus of a different game where the grand coalition has a lower value.

\section{A new game: sequential proposals}

\subsection{The model}

We extend the previous model to $T$ periods where $T$ is assumed to be at least as large as the number of players $n$. The proposer can now make $T$ sequential proposals, and each proposal is answered by the responders as in the previous model. We will denote a generic period as $t$ and a generic stage as $s$. The proposal that emerges at the end of period $t$ and stage $s$ is denoted by $x^{t, s}$, and the proposal that emerges at the end of period $t$ is denoted by $x^{t}:=x^{t, n}$. Given a veto balanced game with a proposer and an order on the set of responders (which may be different for different periods) we will construct an extensive form game, denoted by $G^{T}(N, v)$.

Formally, the resulting outcome of playing the game can be described by the following algorithm.

Input : a veto balanced game $(N, v)$ with a veto player, player 1 , as proposer, and a rule that determines the order of the remaining players (responders). This rule may be different for different periods, and may depend on the history of play. Formally, let $H^{t}$ denote the set of all possible histories of play up to the end of period $t$. For period 1 , the ordering of the responders is a predetermined permutation $\rho^{1}: N \backslash\{1\} \rightarrow N \backslash\{1\}$. For $t>1$, we have a collection of permutations indexed by the history of play in previous periods $\left(\rho_{h^{t-1}}^{t}\right)_{h^{t-1} \in H^{t-1}}$, where $\rho_{h^{t-1}}^{t}: N \backslash\{1\} \rightarrow N \backslash\{1\}$ is the order of the responders in period $t$ given history of play $h^{t-1}$.

Output : a feasible and nonnegative allocation $x$.

1. Start with period 1 . Given a veto balanced TU game $(N, v)$ and the order on the set of responders $\rho^{1}$ corresponding to period 1, players play the game $G(N, v)$. The outcome of this period determines the veto 
balanced TU game for the second period, denoted by $\left(N, v^{2, x^{1}}\right)$, where $v^{2, x^{1}}(S):=\max \left\{0, \min \left\{v(N)-x^{1}(N), v(S)-x^{1}(S)\right\}\right\}$ and $x^{1}$ is the final outcome obtained in the first period. ${ }^{6}$ Note that by construction, the game $\left(N, v^{2, x^{1}}\right)$ is a veto balanced game where player 1 is a veto player. Then go to the next step. The superscripts in the characteristic function denote at which period and after which outcome the game is considered as the game in force. If no confusion arises we write $v^{2}$ instead of $v^{2, x^{1}}$.

2. In period $t(t \leq T)$, given the history of play $h^{t-1}$ and the resulting TU game in place $\left(N, v^{t, x^{t-1}}\right)$, players play the game $G\left(N, v^{t, x^{t-1}}\right)$ with the order of responders determined by $\rho_{h^{t-1}}^{t}$. The outcome of this period determines the veto balanced TU game for the next period, $\left(N, v^{t+1, x^{t}}\right)$, where $v^{t+1, x^{t}}(S):=\max \left\{0, \min \left\{v^{t}(N)-x^{t}(N), v^{t}(S)-x^{t}(S)\right\}\right\}$ and $x^{t}$ is the final outcome obtained in period $t$. Then go to the next step.

3. The game ends after stage $n$ of period $T$. (If at some period before $T$ the proposer makes an efficient proposal (efficient according to the TU game underlying at this period) the game is trivial for the rest of the periods).

4. The outcome is the sum of the outcomes generated at each period, that is, $x:=\sum_{t=1}^{T} x^{t}$.

\subsection{A serial rule for veto balanced games}

We now introduce a solution concept defined on the class of veto balanced games and denoted by $\phi$. Somewhat surprisingly, this solution will be related to the non-cooperative game with sequential proposals.

\footnotetext{
${ }^{6}$ Our definition ensures that $v^{2, x^{1}}(S)$ remains feasible in all subgames, also off the equilibrium path. For example, let $N=\{1,2,3\}, v(1,2)=10, v(1,2,3)=12$ and $v(S)=0$ for all other $S \subseteq N$. If $x^{1}=(1,1,5)$, there has been an agreement on the distribution of a total amount of 7 out of the maximum possible of 12 . The amount that remains to be distributed is $v^{2, x^{1}}(N)=v(N)-x^{1}(N)=12-7=5$. If we calculated $v^{2, x^{1}}(1,2)$ as $v(1,2)-x^{1}(1,2)$ we would obtain $10-2=8$, but this would be infeasible.
} 
Let $(N, v)$ be a veto balanced game where player 1 is a veto player. Define for each player $i$ a value $d_{i}$ as follows:

$$
d_{i}:=\max _{S \subseteq N \backslash\{i\}} v(S) .
$$

Because 1 is a veto player, $d_{1}=0$. Let $d_{n+1}:=v(N)$ and rename the remaining players according to the nondecreasing order of those values. That is, player 2 is the player with the lowest value and so on. The solution $\phi$ associates to each veto balanced game, $(N, v)$, the following payoff vector:

$$
\phi_{l}:=\sum_{i=l}^{n} \frac{d_{i+1}-d_{i}}{i} \text { for all } l \in\{1, \ldots, n\} .
$$

The following example illustrates how the solution behaves.

Example 2 Let $N=\{1,2,3\}$ be a set of players and consider the following 3-person veto balanced game $(N, v)$ where

$$
v(S)=\left\{\begin{array}{cc}
50 & \text { if } S=\{1,2\} \\
10 & \text { if } S=\{1,3\} \\
80 & \text { if } S=N \\
0 & \text { otherwise. }
\end{array}\right.
$$

Computing the vector of $d$-values we get:

$$
\left(d_{1}, d_{2}, d_{3}, d_{4}\right)=(0,10,50,80) .
$$

Applying the formula,

$$
\begin{array}{ll}
\phi_{1}(N, v)=\frac{d_{2}-d_{1}}{1}+\frac{d_{3}-d_{2}}{2}+\frac{d_{4}-d_{3}}{3}=40 \\
\phi_{2}(N, v)= \\
\phi_{3}(N, v)= & \frac{d_{3}-d_{2}}{2}+\frac{d_{4}-d_{3}}{3}=30 \\
\frac{d_{4}-d_{3}}{3} & =10
\end{array}
$$

The formula suggests a serial rule principle (cf. Moulin and Shenker, 1992). Since it is not possible for any coalition to obtain a payoff above $d_{i}$ without player $i$ 's cooperation, we can view player $i$ as having a right over 
the amount $v(N)-d_{i}$. The value $v(N)$ is divided into segments $\left(d_{2}-d_{1}\right.$, $\left.d_{3}-d_{2}, \ldots, v(N)-d_{n}\right)$ and each payoff segment is divided equally among the players that have a right over it.

In the class of veto balanced games, the solution $\phi$ satisfies some wellknown properties such as nonemptiness, efficiency, anonymity and equal treatment of equals among others. It also satisfies aggregate monotonicity. ${ }^{7}$

The next section shows that $\phi(N, v)$ is the unique equilibrium outcome assuming that all responders act as myopic maximizers and the proposer plays optimally taking this into account.

\subsection{Myopic Best Response Equilibrium}

We start our analysis of the non-cooperative game with sequential proposals by assuming myopic behavior on the part of responders. Responders behave myopically when they act as payoff maximizers in each period without considering the effect of their actions on future periods.

Suppose all responders maximize payoffs myopically for each period and that the proposer plays optimally taking into account that the responders are myopic maximizers. Formally, player $i \neq 1$ maximizes $x_{i}^{t}$ at each period $t$ whereas player 1 maximizes $\sum_{t=1}^{T} x_{1}^{t}$. We call such a strategy profile a myopic best response equilibrium (MBRE). We will show in this section that all MBRE lead to the same outcome, namely the serial rule.

In order to show this result, we introduce the concept of balanced proposals, which are proposals that emerge as the final outcome regardless of whether they are accepted or rejected by the responders. We then show that any payoff the proposer can attain under myopic behavior of the responders can also be attained by making balanced proposals: player 1 can cut the payoff of other players until a balanced proposal is obtained at no cost to himself (lemma 4). Hence, from the proposer's point of view there is no loss of generality in restricting the analysis to balanced proposals. We will then

\footnotetext{
${ }^{7}$ For a definition of those properties, see Peleg and Sudhölter (2003). It is not the aim of this paper to provide an axiomatic analysis of the solution. Arin and Feltkamp (2012) characterize the solution in the domain of veto balanced games by core selection and a monotonicity property.
} 
show that the highest payoff the proposer can achieve with balanced proposals is $\phi_{1}$. Finally, we will show that the only way in which the proposer can achieve $\phi_{1}$ requires all players to get their component of the serial rule, so that the only MBRE outcome is $\phi(N, v)$.

Note that the property of balancedness in a proposal holds or fails to hold for all possible orders of the responders. Because of this, the results in this section hold for any order of moves of the responders.

Our main motivation for the use of MBRE is its simplicity. Beyond that, it can be justified as modelling cautious behavior on the part of the responders. If the responders are not certain of other players' rationality, they may choose to maximize payoffs for the current period without trying to anticipate other players' future behavior. What if we assume similarly cautious behavior on the part of the proposer? This would lead us to the concept of balanced proposals. Hence, in a MBRE with balanced proposals all players are playing cautiously. Our results on MBRE and balanced proposals indicate that it is enough that one of the sides (proposer or responders) is acting cautiously in order to obtain the serial rule.

\subsubsection{Balanced proposals}

The notion of balanced proposals will play a central role in the analysis of MBRE.

Definition 1 Let $(N, v)$ be a veto balanced $T U$ game, and $G^{T}(N, v)$ its associated extensive form game. Given a period $t$, a proposal $x$ is balanced if it is the final outcome of period $t$ regardless of the actions of the responders.

Balanced proposals coincide with the nucleolus (kernel) of special games. In the class of veto-rich games (games with at least one veto player and a nonempty set of imputations) the kernel and the nucleolus coincide (Arin and Feltkamp, 1997). Therefore we can define the nucleolus as

$$
\nu(N, v):=\left\{x \in I(N, v): f_{i j}(x)<f_{j i}(x) \Longrightarrow x_{j}=0\right\}
$$

We use this alternative definition of the nucleolus in the proof of the following lemma. 
Lemma 2 Let $(N, v)$ be a veto balanced TU game. Consider the associated game $G^{T}(N, v)$. Given a period $t$, a proposal $x^{t}$ is balanced if and only if it coincides with the nucleolus of the game $\left(N, w^{t}\right)$, where $w^{t}(S)=v^{t}(S)$ for all $S \neq N$ and $w^{t}(N)=x^{t}(N)$.

Proof. Assume that $x^{t}$ is a balanced proposal in period $t$ with the game $\left(N, v^{t}\right)$.

a) Let $l$ be a responder for which $x_{l}^{t}=0$. If whatever the response of player $l$ the proposal does not change then $f_{1 l}\left(x^{t}\right) \leq 0=x_{l}^{t}=f_{l 1}\left(x^{t}\right)$.

b) Let $m$ be a responder for which $x_{m}^{t}>0$. If whatever the response of player $m$ the proposal does not change then $f_{1 m}\left(x^{t}\right)=x_{m}^{t}=f_{m 1}\left(x^{t}\right)$.

Therefore, the bilateral kernel conditions are satisfied for the veto player. Lemma 12 in Arin and Feltkamp (2007) shows that if the bilateral kernel conditions are satisfied between the veto player and the rest of the players then the bilateral kernel conditions are satisfied between any pair of players.

Therefore, $x^{t}$ is the kernel (nucleolus) of the game $\left(N, w^{t}\right)$. The converse statement can be proven in the same way.

\subsubsection{Balanced proposals and MBRE}

If there is only one period in the game, myopic and rational behavior coincide. This means that the following lemma holds if responders behave myopically.

Lemma 3 (Arin and Feltkamp, 2007, lemmas 2 and 3) Let $(N, v)$ be a veto balanced TU game, and $G^{T}(N, v)$ its associated extensive form game. At any period $t$ and stage $s$, the responder (say, i) will accept $x^{t, s-1}$ if $f_{i 1}\left(x^{t, s-1}, v^{t}\right)>$ $f_{1 i}\left(x^{t, s-1}, v^{t}\right)$, and will reject it if $f_{i 1}\left(x^{t, s-1}, v^{t}\right)<f_{1 i}\left(x^{t, s-1}, v^{t}\right)$ in a MBRE. If $f_{i 1}\left(x^{t, s-1}, v^{t}\right)=f_{1 i}\left(x^{t, s-1}, v^{t}\right)$, the responder is indifferent between accepting and rejecting since both decisions lead to the same outcome. Also, the final outcome $x^{t}$ of any period $t$ is such that $f_{i 1}\left(x^{t}, v^{t}\right) \geq f_{1 i}\left(x^{t}, v^{t}\right)$ for all $i$.

We have established that myopic behavior of the responders leads to $f_{i 1}\left(x^{t}, v^{t}\right) \geq f_{1 i}\left(x^{t}, v^{t}\right)$, or equivalently to $x_{i}^{t} \geq f_{1 i}\left(x^{t}, v^{t}\right)$. We now show that the proposer can obtain the same payoff with balanced proposals in all such cases. 
Lemma 4 Let $(N, v)$ be a veto balanced game. Consider the associated game with $T$ periods $G^{T}(N, v)$. Let $z=\sum_{t=1}^{T} x^{t}$ be the outcome resulting from an arbitrary strategy profile. Assume that the final outcome of any period $t, x^{t}$, is such that for any player $i, x_{i}^{t} \geq f_{1 i}\left(x^{t}, v^{t}\right)$. Then there exists $y$ such that $y_{1}=z_{1}, y=\sum_{t=1}^{T} q^{t}$ where $q^{t}$ is a balanced proposal for period $t$.

Proof. If $\left(x^{1}, x^{2}, \ldots, x^{T}\right)$ is a sequence of balanced proposals the proof is done.

Suppose that $\left(x^{1}, x^{2}, \ldots, x^{T}\right)$ is not a sequence of balanced proposals. This means that for some $x^{t}$ and for some $i \neq 1$ it holds that $x_{i}^{t}>f_{1 i}\left(x^{t}, v^{t}\right)$ and $x_{i}^{t}>0$. Let $k$ be the first period where such result holds. Therefore, $\left(x^{1}, x^{2}, \ldots, x^{k-1}\right)$ is a sequence of balanced proposals. We will construct a balanced proposal where the payoff of the proposer does not change.

Since $f_{i 1}\left(x^{k}\right)=x_{i}^{k}$, by reducing the payoff of player $i$ we can construct a new allocation $y^{k}$ such that $f_{1 i}\left(y^{k}\right)=f_{i 1}\left(y^{k}\right)$ or $f_{1 i}\left(y^{k}\right)<f_{i 1}\left(y^{k}\right)$ and $y_{i}^{k}=0$. In any case, $x_{1}^{k}=y_{1}^{k}$ and the payoff of the proposer does not change. Note also that reducing $i$ 's payoff cannot increase $f_{1 j}\left(y^{k}\right)$, so it is still the case that $f_{1 j}\left(y^{k}\right) \leq f_{j 1}\left(y^{k}\right)$ for all $j$.

Now, if there exists another player $l$ such that $f_{1 l}\left(y^{k}\right)<f_{l 1}\left(y^{k}\right)$ and $y_{l}^{k}>0$ we construct a new allocation $z^{k}$ such that $f_{1 l}\left(z^{k}\right)=f_{l 1}\left(z^{k}\right)$ or $f_{1 i}\left(z^{k}\right)<f_{i 1}\left(z^{k}\right)$ and $z_{i}^{k}=0$. Note that $z_{1}^{k}=y_{1}^{k}$. Repeating this procedure we will end up with a balanced allocation $q^{k}$.

The TU game $\left(N, w^{k+1}\right)$ resulting after proposing $q^{k}$ satisfies that $w^{k+1}(S) \geq$ $v^{k+1}(S)$ for all $S \ni 1$. Therefore, $f_{1 i}\left(x^{k+1}, w^{k+1}\right) \leq f_{1 i}\left(x^{k+1}, v^{k+1}\right)$, and $x_{l}^{k+1} \geq f_{1 l}\left(x^{k+1}, w^{k+1}\right)$ for all $l$.

Consider the game $\left(N, w^{k+1}\right)$ and the payoff $x^{k+1}$. Suppose that $x_{i}^{k+1}>$ $f_{1 i}\left(x^{k+1}\right)$ for some $i \neq 1$ and $x_{i}^{k+1}>0$. We can repeat the same procedure of period $k$ until we obtain a balanced allocation $q^{k+1}$. The procedure can be repeated until the last period of the game to obtain the sequence of balanced proposals $\left(x^{1}, x^{2}, \ldots, x^{k-1}, q^{k}, \ldots, q^{T}\right)$.

Corollary 1 A proposer's payoff $z_{1}$ is achievable under myopic behavior of the responders if and only if it is achievable by balanced proposals. 
If responders behave myopically, the proposer has a sequence of balanced proposals that yields the same proposer's payoff (lemmas 3 and 4). The converse statement is trivial: if a payoff is achievable by balanced proposals, it is achievable under any assumptions about responders' behavior including myopic behavior.

\subsubsection{The serial rule can be achieved with balanced proposals}

We now show that, by making balanced proposals, the proposer can secure the payoff provided by the serial rule $\phi$. Note that the assumption $T \geq n$ is crucial for this result.

Lemma 5 Let $(N, v)$ be a veto balanced $T U$ game and $G^{T}(N, v)$ its associated extensive form game with $T \geq n$. The proposer has a sequence of balanced proposals that leads to $\phi(N, v)$.

Proof. The sequence consists of $n$ balanced proposals. At each period $t,(t \in\{1, \ldots, n\})$ consider the set $S_{t}=\{l: l \leq t\}$ and the proposal $x^{t}$, defined as follows:

$$
x_{l}^{t}=\left\{\begin{array}{cc}
\frac{d_{t+1}-d_{t}}{t} & \text { for all } l \in S_{t} \\
0 & \text { otherwise. }
\end{array}\right.
$$

whenever $x^{t}$ is feasible and propose the 0 vector otherwise.

It can be checked immediately that in each period the proposed allocation will be the final allocation independently of the answers of the responders and independently of the order of those answers. The proposals are balanced proposals.

For example, in period 1 , the proposal is $\left(d_{2}, 0, \ldots, 0\right)$. Because 1 is a veto player, $f_{i 1}()=$.0 for all $i$. As for $f_{1 i}($.$) , because all players other than 1$ are getting 0 , the coalition of minimum satisfaction of 1 against $i$ is also the coalition of maximum $v(S)$ with $i \notin S$. Call this coalition $S^{*}$. By definition, $v\left(S^{*}\right)=d_{i} \geq d_{2}$ and $f_{1 i}()=.x\left(S^{*}\right)-v\left(S^{*}\right)=d_{2}-d_{i} \leq 0$. Thus, $f_{i 1}(.) \geq f_{1 i}($. for all $i$ and the outcome of period 1 is $\left(d_{2}, 0, \ldots, 0\right)$ regardless of responders' behavior.

In period 2 we have a game $v^{2}$ with the property that $v^{2}(S)>0$ implies $v^{2}(S)=v^{1}(S)-d_{2}$ for all $S$. Thus, player 2 is a veto player in $v^{2}$. Player 1 
proposes $\left(\frac{d_{3}-d_{2}}{2}, \frac{d_{3}-d_{2}}{2}, 0, \ldots, 0\right)$. If player 2 rejects, we have $f_{12}()=.\frac{d_{3}-d_{2}}{2}-$ $0=f_{21}($.$) . As for other players i \neq 1,2$, when computing $f_{1 i}$ we take into account that any coalition of positive value must include 1 and 2 . Since players other than 1 and 2 are getting 0 , the coalition 1 uses against $i$ is $S^{*} \in \arg \max _{S: i \notin S} v(S)$. By definition, $v\left(S^{*}\right)=d_{i}$ and $v^{2}\left(S^{*}\right)=d_{i}-d_{2}$. Then $f_{1 i}()=.x\left(S^{*}\right)-v^{2}\left(S^{*}\right)=\left(d_{3}-d_{2}\right)-\left(d_{i}-d_{2}\right)=d_{3}-d_{i} \leq 0$.

In period 3, player 3 has become a veto player and the same process can be iterated until period $n$.

Therefore, this strategy of the proposer determines the total payoff of all the players, that is, the final outcome of the game $G^{T}(N, v)$. This final outcome coincides with the solution $\phi$.

Remark 1 The serial rule can also be obtained by making balanced proposals if the game has $n-1$ periods.

This is because the proposer can combine the first two proposals in the proof of lemma 5 by proposing $\left(d_{2}+\frac{d_{3}-d_{2}}{2}, \frac{d_{3}-d_{2}}{2}, 0, \ldots, 0\right)$ in the first period.

The proof of lemma 5, together with lemma 2, suggests a new interpretation of the serial rule. At each period the proposal coincides with the nucleolus of a veto-rich game. Formally,

Remark 2 The serial rule is the sum of the nucleolus allocations of $n$ auxiliary games, namely

$$
\phi(N, v)=\sum_{i=1}^{n} \nu\left(N, w^{i}\right)
$$

where the games $\left(N, w^{t}\right)$ are defined as follows: $w^{1}(N)=d_{2}$ and $w^{1}(S)=$ $v(S)$ otherwise. For $i: 2, . ., n$ :

$$
w^{i}(S):=\left\{\begin{array}{cc}
d_{i+1}-d_{i} & \text { if } S=N \\
\max \left\{0, w^{i-1}(S)-\sum_{l \in S} \nu_{l}\left(N, w^{i-1}\right)\right\} & \text { otherwise. }
\end{array} .\right.
$$

This interpretation of the serial rule provides a better understanding of the connection between the serial rule and the model with sequential proposals. In the one-period game the kernel (nucleolus) of the veto game is a 
natural candidate to be a Nash outcome of the noncooperative model since it satisfies the bilateral principle that is applied in the event of disagreement; in many cases this intuition is confirmed (Arin and Feltkamp, 2007). In contrast, the model with $n$ periods leads to the serial rule, an apparently unrelated solution concept. However, since the nucleolus of an auxiliary game is obtained in each period, the outcome of the $n$-period game is not incongruent with the outcome of the one-period game. ${ }^{8}$

\subsubsection{The serial rule is the only MBRE outcome}

Theorem 2 Let $(N, v)$ be a veto balanced $T U$ game and $G^{T}(N, v)$ the associated extensive form game with $T \geq n$. Let $z=\sum_{t=1}^{T} x^{t}$ be an outcome resulting from a MBRE of $G^{T}(N, v)$. Then $z=\phi(N, v)$.

Proof. We have already shown that the proposer can achieve $\phi_{1}(N, v)$ with balanced proposals. It remains to show that the proposer cannot improve upon $\phi$. Let $z=\sum_{t=1}^{T} x^{t}$ be an outcome resulting from balanced proposals. The strategy of the proof is to show that $z_{1} \geq \phi_{1}$ implies $z_{i} \geq \phi_{i}$ for all $i$. This result, together with the efficiency of the serial rule, leads to $z=\phi$ being the unique MBRE outcome. See Appendix for details.

As a byproduct of the analysis, we are able to compare the serial rule and the nucleolus from player 1's point of view.

Corollary 2 Let $(N, v)$ be a veto balanced $T U$ game. Then $\phi_{1}(N, v) \geq$ $\nu_{1}(N, v)$.

Proof. As we have seen, $\phi_{1}(N, v)$ coincides with the MBRE payoff for the proposer in the game $G^{T}(N, v)$ when $T \geq n-1$. This payoff is at least as large as his equilibrium payoff in the game $G^{1}(N, v)$, because the proposer can always wait until period $T$ to divide the payoff. This equilibrium payoff

\footnotetext{
${ }^{8}$ Arin and Katsev (2014) show that in the class of veto balanced games the serial rule coincides with the SD-Prenucleolus, a solution concept defined in the class of all TU games that has similarities with the per capita prenucleolus and the prenucleolus. This result reinforces the unexpected relationship between two apparently very different concepts.
} 
is in turn at least as high as $\nu_{1}(N, v)$, because $\nu(N, v)$ is a balanced proposal.

\subsection{MBRE and SPE may not coincide}

The next example illustrates that a MBRE need not be a subgame perfect equilibrium.

Example 3 Let $N=\{1,2,3,4,5\}$ be the set of players and consider the following 5-person veto balanced game $(N, v)$ where

$$
v(S)=\left\{\begin{array}{cc}
36 & \text { if } S \in\{\{1,2,3,4\},\{1,2,3,5\}\} \\
31 & \text { if } S=\{1,2,4,5\} \\
51 & \text { if } S=N \\
0 & \text { otherwise. }
\end{array}\right.
$$

The serial rule for this game can be easily calculated given that $d_{1}=d_{2}=$ $0, d_{3}=31, d_{4}=d_{5}=36$ and $d_{6}=51$. Player 1's payoff according to the serial rule is then $\phi_{1}(N, v)=\frac{31}{2}+\frac{36-31}{3}+\frac{51-36}{5}=121 / 6$. As we know from the previous section, this is player 1's payoff in any MBRE for any order of the responders. Suppose the order of responders is 2, 3, 4, 5. The following result holds given this order: If the responders play the game optimally (not necessarily as myopic maximizers) the proposer can get a higher payoff than the one provided by the MBRE outcome. Therefore, MBRE and SPE outcomes do not necessarily coincide.

The strategy is the following: The proposer offers nothing in the first three periods. In the 4 th period the proposal is: $(10,10,5,0,0)$.

The responses of players 2, 4 and 5 do not change the proposal (even if the proposal faced by player 4 and 5 is a new one resulting from a rejection of player 3). If player 3 accepts this proposal, the TU game for the last period will be:

$$
w(S)=\left\{\begin{array}{cc}
11 & \text { if } S \in\{\{1,2,3,4\},\{1,2,3,5\}\} \\
11 & \text { if } S=\{1,2,4,5\} \\
26 & \text { if } S=N \\
0 & \text { otherwise. }
\end{array}\right.
$$


In the last period, myopic and rational behavior coincide, so the outcome must be an element of $B_{1}(N, w)$. It can be checked that $B_{1}(N, w)=$ $\{(5.5,5.5,0,0,0)\}$. Therefore, after accepting the proposal in period 4 , player 3 gets a total payoff of 5 .

If player 3 rejects the proposal, the outcome of the 4 th period is $(15,10,0,0,0)$ and the TU game for the last period is:

$$
u(S)=\left\{\begin{array}{cc}
11 & \text { if } S \in\{\{1,2,3,4\},\{1,2,3,5\}\} \\
6 & \text { if } S=\{1,2,4,5\} \\
26 & \text { if } S=N \\
0 & \text { otherwise. }
\end{array}\right.
$$

As before, in the last period myopic and rational behavior coincide and the outcome must be an element of $B_{1}(N, u)$. It can be checked that $B_{1}(N, u)=$ $\{(5.2,5.2,5.2,5.2,5.2)\}$. Therefore, after rejecting the proposal player 3 gets a total payoff of 5.2 .

Therefore, rational behavior of player 3 implies a rejection of the proposal in the 4th period. This rejection is not a myopic maximizer's behavior. After the rejection of player 3 the proposer gets a payoff of 20.2 , higher than $121 / 6$. Hence, the outcome associated to MBRE is not the outcome of an SPE.

In the example above, the proposer finds a credible way to collude with player 3 in order to get a higher payoff than the one obtained by player 2 (a veto player). Player 2 cannot avoid this collusion since he is responding before player 3 . If he responded after player 3, collusion between players 1 and 3 would no longer be profitable. This observation turns out to be crucial as we will see in the next section.

\subsection{The serial rule as an SPE outcome}

The previous example shows that, in general, myopic and rational behavior do not coincide. However, they do coincide when the model incorporates a requirement on the order of the responders. We will assume in theorem 3 that stronger responders must move after weaker responders. Formally, denote the $d$-value for player $i$ in $v^{t}$ as $d_{i}^{t}:=\max _{S \subseteq N \backslash\{i\}} v^{t}(S)$. We assume that responders move following the order of nonincreasing $d$-values of $v^{t}$, that is, 
that player $j$ moving earlier than player $k$ at time $t$ implies $d_{j}^{t} \geq d_{k}^{t} \cdot{ }^{9}$ Given this order, any veto responder can secure a payoff equal to the one obtained by the proposer. This was not the case in Example 3, where player 2 is a veto responder responding before player 3 .

Theorem 3 Let $(N, v)$ be a veto balanced TU game and $G^{T}(N, v)$ its associated extensive form game in which $T \geq n$ and the responders move following the order of nonincreasing $d$-values of $v^{t}$. Then $\phi(N, v)$ is the outcome of any $S P E$.

Proof. (See Appendix for details) The strategy of the proof is to show that, if responders move following the order of nonincreasing $d$-values of $v^{t}$, any SPE outcome $z$ is such that the proposer can obtain $z_{1}$ by making balanced proposals. Since the best outcome achievable by balanced proposals is $\phi(N, v)$ as shown in section 3.3, this will complete the proof.

\section{Concluding remarks}

We have studied a bargaining procedure with a distinguished player and an enforceable bilateral principle in case of disagreement. The nucleolus is the only efficient allocation that satisfies this bilateral principle, but it does not always emerge as an equilibrium outcome because the proposer may prefer to make an inefficient proposal. We then introduced the possibility of renegotiation through additional periods of bargaining and showed that it leads to an efficient outcome, namely the serial rule. As a byproduct of the analysis, we uncovered a relationship between the serial rule and the nucleolus: the serial rule is the sum of $n$ allocations, each of which is the nucleolus of an auxiliary game.

We have shown that any SPE outcome of our bargaining procedure is achievable with myopic behavior of the responders if responders move by increasing strength (lemma 14 in the Appendix). This result is independent of the number of periods. If there are at least $n-1$ periods, the only SPE

\footnotetext{
${ }^{9}$ Note that we do not rule out $d_{j}^{t}=d_{k}^{t}$, in which case there is more than one order of moves compatible with this condition. Theorem 3 holds for any such order of moves.
} 
outcome is the serial rule: the proposer is always able to obtain $\phi_{1}(N, v)$ by making balanced proposals, and the only way to obtain this payoff is if all other players get $\phi_{i}(N, v)$ as well. If there are fewer than $n-1$ periods, there may not be enough periods for the proposer to achieve the serial rule with balanced proposals. If $z$ is an SPE outcome, it is still true that the proposer can obtain $z_{1}$ by making balanced proposals, hence all SPE outcomes must have the same $z_{1}$, but there may be several SPE outcomes if $z_{1}<\phi_{1}(N, v)$.

Our paper is closely related to Dagan et al. (1997) in that both papers are based on bilateral bargaining with a certain bilateral principle being enforced in the event of disagreement. Nevertheless, there is a fundamental difference with this and other papers (e.g. Chun (1989), Herrero et al. (2010) and Karagözoğlu (2014)) that makes it difficult to relate our paper to the literature on noncooperative models of bankruptcy: our bargaining procedure does not feature an exogenous vector of claims $\left(c_{i}\right)_{i \in N}$. The values $v(N)-$ $d_{i}$ could be considered analogous to claims, but note that they are not an exogenous element of the model (they just happen to play an important role in equilibrium) and there is no suggestion that player $i$ is entitled to receive the entire $v(N)-d_{i}$, just that achieving a payoff above $d_{i}$ would require player $i$ 's consent so that player $i$ has a veto right over $v(N)-d_{i}$. However, it is worth noting that, if we define $c_{i}:=v(N)-d_{i}$, the serial rule coincides with Ibn Ezra's solution as described by O'Neill (1982).

\section{Appendix}

\subsection{Proof of theorem 2}

We have already shown that $\phi(N, v)$ can be achieved with balanced proposals. It remains to show that the proposer cannot improve upon $\phi$. Let $z=\sum_{t=1}^{T} x^{t}$ be an outcome resulting from balanced proposals. Our objective is to show that $z_{1} \geq \phi_{1}$ implies $z_{i} \geq \phi_{i}$ for all $i$. This result, together with the efficiency of the serial rule, leads to $z=\phi$ being the unique MBRE outcome. We start by establishing the result not for the original game $(N, v)$, but for the sequence of auxiliary games $\left(N, w^{t}\right)$ (lemma 9$)$. We then check that the sum 
of the serial rules of the games $w^{t}$ cannot exceed the serial rule of the original game $(N, v)$ (lemma 10).

We start with the following property of balanced proposals:

Lemma 6 If $x^{t}$ is a balanced proposal, any player $i$ with $x_{i}^{t}>0$ will be a veto player at $t+1$.

This is because if $x^{t}$ is balanced we have $f_{1 i}\left(x^{t}, v^{t}\right)=x_{i}^{t}$, so that all coalitions that have a positive $v^{t}$ but do not involve $i$ have $v^{t}(S)<x^{t}(S)$. Thus, after the payoffs $x^{t}$ are distributed any coalition with positive value must involve $i$.

The following lemma establishes a relationship between balanced proposals in $G^{T}(N, v)$ and the serial rule. Suppose $x^{t}$ is a balanced proposal in period $t$. Consider the game $w^{t}$, where $w^{t}(S)=\min \left\{v^{t}(S), x^{t}(N)\right\}$. The serial rule of $w^{t}$ and the balanced proposal $x^{t}$ do not coincide in general. However, the set of players who receive a positive payoff in $x^{t}$ coincides with the set of players who receive a positive payoff according to the serial rule of $w^{t} .10$

Lemma 7 Let $(N, v)$ be a veto balanced TU game. Consider the associated game $G^{T}(N, v)$. Let $z=\sum_{t=1}^{T} x^{t}$ be the outcome resulting from some strategy profile with balanced proposals. Consider period $t$, its outcome $x^{t}$ and the game $\left(N, w^{t}\right)$ where $w^{t}(S)=\min \left\{v^{t}(S), x^{t}(N)\right\}$. Then it holds that $x_{k}^{t}>0$ if and only if $\phi_{k}\left(N, w^{t}\right)>0$.

Proof. a) If $x_{k}^{t}>0$ we need to prove that $d_{k}\left(N, w^{t}\right)<x^{t}(N)$, so that the serial rule of $w^{t}$ assigns a positive payoff to $k$.

Let $S \in \arg \max _{T \subseteq N \backslash\{k\}} v^{t}(T)$. Since $x^{t}$ is balanced we have $f_{1 k}\left(x^{t}\right)=$ $x_{k}^{t}>0$ and that implies $x^{t}(S)>v^{t}(S)$ (otherwise $S$ could have been used

\footnotetext{
${ }^{10}$ For example, consider the game with $N=\{1,2,3,4\}, v(1,2)=v(1,3)=2, v(1,2,3)=$ $6, v(1,2,3,4)=10$ and $v(S)=0$ otherwise. The proposal $x=(2,1.5,1.5,0)$ is a balanced proposal with a total payoff distributed of 5 (and, because of lemma 2 and the uniqueness of the nucleolus, it is the only balanced proposal that distributes a total payoff of 5 ). The game $w$ associated to this proposal is identical to $v$ except that $w(1,2,3)=w(N)=5$. Its serial rule is $(3,1,1,0)$, which is different from the balanced proposal but gives a positive payoff to the same set of players.
} 
to complain against $k$ ). Hence, $x^{t}(N) \geq x^{t}(S)>v^{t}(S)=d_{k}\left(v^{t}\right)=d_{k}\left(w^{t}\right)$, where the last equality follows from lemma $6 .{ }^{11}$

b) If $x_{k}^{t}=0$ we need to prove that $d_{k}\left(N, w^{t}\right)=x^{t}(N)$. Since $x^{t}$ is balanced, $f_{1 k}\left(x^{t}, v^{t}\right) \leq 0$. Let $P$ be a coalition associated to $f_{1 k}\left(x^{t}, v^{t}\right)$. Because $f_{1 k}\left(x^{t}, v^{t}\right) \leq 0, x^{t}(P) \leq v^{t}(P)$. Coalition $P$ must contain all players receiving a positive payoff at $x^{t}$ (otherwise $x^{t}$ is not balanced since $P$ can be used against any player outside $P$ ). Therefore $x^{t}(N)=x^{t}(P) \leq v^{t}(P)$. Because of the way $w^{t}$ is defined it cannot exceed $x^{t}(N)$, so $x^{t}(N)=w^{t}(P)=$ $d_{k}\left(w^{t}\right)$ and $k$ receives 0 according to the serial rule of $w^{t}$.

The following lemma concerns a property of the serial rule. By definition, the serial rule is such that $d_{k}$ is divided among players $\{j \in N, j<k\}$. Above $d_{k}$, player $k$ and any player $j<k$ get the same payoff.

Lemma 8 For any player $k$ we have $\sum_{i \in\{1,2, \ldots, k-1\}} \phi_{i}=d_{k}+(k-1) \phi_{k}$. Hence, $\sum_{i \in\{1,2, \ldots, k-1\}} \phi_{i} \geq d_{k}+\phi_{k}$. The latter inequality is strict except if $k=2$ or $\phi_{k}=0$.

The next lemma tell us that, given a strategy profile with balanced proposals, the proposer cannot get more than the serial rule of the games $w^{t}$.

Lemma 9 Let $(N, v)$ be a veto balanced TU game. Consider the associated game with $T$ periods $G^{T}(N, v)$. Let $z=\sum_{t=1}^{T} x^{t}$ be an outcome resulting from balanced proposals. Consider period $t$, its outcome $x^{t}$ and the game $\left(N, w^{t}\right)$ where $w^{t}(S)=\min \left\{v^{t}(S), x^{t}(N)\right\}$. Then $x_{1}^{t} \geq \phi_{1}\left(N, w^{t}\right)$ implies $x_{l}^{t} \geq \phi_{l}\left(N, w^{t}\right)$ for all $l \in N$.

Proof. Let $T$ be the set of veto players in $\left(N, w^{t}\right)$, and let $M=\left\{l_{1}, \ldots, l_{m}\right\}$ be the ordered (according to the $d$-values of $\left(N, w^{t}\right)$ ) set of nonveto players that have received a positive payoff at $x^{t}$. That is, $d_{l_{1}} \leq \ldots \leq d_{l_{m}}{ }^{12}$

\footnotetext{
${ }^{11}$ Because $x^{t}$ is a balanced proposal, the $d$-values of $w^{t}$ coincide with the $d$-values of $v^{t}$ for all players receiving a positive payoff. Any player $j$ that is receiving a positive payoff at $t$ will be veto at $t+1$ (lemma 6$)$. The values $d_{j}\left(w^{t}\right)$ and $d_{j}\left(v^{t}\right)$ can only differ if $v^{t}(S)>x^{t}(N)$ for some $S$ such that $j \notin S$, but then player $j$ would not be veto at $t+1$.

${ }^{12}$ Recall that, because $x^{t}$ is a balanced proposal, the $d$-values of $w^{t}$ coincide with the $d$-values of $v^{t}$ for all players in $M$.
} 
Suppose $x_{1}^{t} \geq \phi_{1}\left(N, w^{t}\right)$. Since $x^{t}$ is balanced, $x_{1}^{t}=x_{i}^{t}$ for all $i \in T$, thus if $x_{1}^{t} \geq \phi_{1}\left(N, w^{t}\right)$ it follows that $x_{i}^{t} \geq \phi_{i}\left(N, w^{t}\right)$ for all $i \in T$.

We now want to prove that $x_{i}^{t} \geq \phi_{i}\left(N, w^{t}\right)$ for all $i \in M$. We will do it by induction.

Consider the responder $l_{1}$. Since $x^{t}$ is balanced we have $f_{1 l_{1}}\left(x^{t}\right)=x_{l_{1}}^{t}$. If the coalition associated to $f_{1 l_{1}}$ has a value of 0 , it follows that $x_{1}^{t}=x_{l_{1}}^{t}$ so $x_{l_{1}}^{t} \geq \phi_{l_{1}}\left(N, w^{t}\right)$. If the coalition 1 is using has a positive value, all veto players must be in it, so its payoff must be at least $|T| \phi_{1}\left(N, w^{t}\right)$, and its value (by definition of $d_{l_{1}}$ ) cannot exceed $d_{l_{1}}$. Hence, $f_{1 l_{1}}\left(x^{t}\right) \geq|T| \phi_{1}\left(N, w^{t}\right)-d_{l_{1}}$. Because of lemma 8, $|T| \phi_{1}\left(N, w^{t}\right)-d_{l_{1}} \geq \phi_{l_{1}}\left(N, w^{t}\right)$.

Now suppose the result $x_{i}^{t} \geq \phi_{i}\left(N, w^{t}\right)$ is true for all $i \in\left\{l_{1}, \ldots, l_{k-1}\right\}$. We will prove that $x_{l_{k}}^{t} \geq \phi_{l_{k}}\left(N, w^{t}\right)$. Let $S$ be a coalition such that $f_{1 l_{k}}\left(x^{t}\right)=$ $x^{t}(S)-v^{t}(S)$. As before, the result follows immediately if $v^{t}(S)=0$. If $v^{t}(S)>0$ it must be the case that $T \subseteq S$, but $S$ need not involve all players in $\left\{l_{1}, \ldots, l_{k-1}\right\}$. Denote $\left\{l_{1}, \ldots, l_{k-1}\right\}$ by $Q$. We consider two cases, depending on whether $Q \subseteq S$.

If $Q \subseteq S$, we have $x_{l_{k}}^{t}=f_{1 l_{k}}\left(x^{t}\right)=x^{t}(S)-v^{t}(S) \geq \sum_{i \in T \cup Q} \phi_{i}\left(N, w^{t}\right)-d_{l_{k}}$, where the last inequality uses the induction hypothesis. The set $T \cup Q$ contains all players with $d_{i}<d_{l_{k}}$. Hence, by lemma 8, $\sum_{i \in T \cup Q} \phi_{i}\left(N, w^{t}\right)-$ $d_{l_{k}} \geq \phi_{l_{k}}\left(N, w^{t}\right)$.

If $Q \nsubseteq S$, there is a player $l_{p}<l_{k}$ such that $l_{p} \notin S$. Because $x^{t}$ is a balanced proposal, $x_{l_{p}}^{t}=f_{1 l_{p}}\left(x^{t}\right)$. Because the veto player can use $S$ to complain against $l_{p}, f_{1 l_{p}}\left(x^{t}\right) \leq f_{1 l_{k}}\left(x^{t}\right)=x_{l_{k}}$, hence $x_{l_{p}} \leq x_{l_{k}}$. By the induction hypothesis, $x_{l_{p}} \geq \phi_{l_{p}}\left(N, w^{t}\right)$. Since $d_{l_{p}} \leq d_{l_{k}}$ we also know that $\phi_{l_{p}}\left(N, w^{t}\right) \geq \phi_{l_{k}}\left(N, w^{t}\right)$, so that

$$
x_{l_{k}}=f_{1 l_{k}}\left(x^{t}\right) \geq f_{1 l_{p}}\left(x^{t}\right)=x_{l_{p}} \geq \phi_{l_{p}}\left(N, w^{t}\right) \geq \phi_{l_{k}}\left(N, w^{t}\right) .
$$

So far we have discussed the set of veto players and the set of nonveto players that are getting a positive payoff in $x^{t}$. For players in $N \backslash(T \cup M)$, we have shown in lemma 7 that $x_{j}^{t}=0$ implies $\phi_{j}\left(N, w^{t}\right)=0$, hence $x_{j}^{t} \geq$ $\phi_{j}\left(N, w^{t}\right)$ for all players.

Corollary 3 If $z=\sum_{t=1}^{T} x^{t}$ is an outcome resulting from balanced proposals, $x_{1}^{t} \geq \phi_{1}\left(N, w^{t}\right)$ implies $x_{l}^{t}=\phi_{l}\left(N, w^{t}\right)$ for all $l \in N$. 
This corollary follows directly from lemma 9 and the efficiency of the serial rule. Lemma 9 states that $x_{1}^{t} \geq \phi_{1}\left(N, w^{t}\right)$ implies $x_{l}^{t} \geq \phi_{l}\left(N, w^{t}\right)$ for all $l \in N$. By definition of $w^{t}, \sum_{l \in N} x_{l}^{t}=w^{t}(N)$. By the efficiency of the serial rule, $\sum_{l \in N} \phi_{l}\left(N, w^{t}\right)=w^{t}(N)$. Hence, the only way in which player 1 can obtain the serial rule of $\left(N, w^{t}\right)$ with balanced proposals is that all players in the game obtain their serial rule payoff.

Finally, the sum of the serial rules of the games $w^{t}$ cannot exceed the serial rule of the original game. This is due to the following property of the serial rule:

Lemma 10 Consider the veto balanced TU game $(N, v)$ and a finite set of positive numbers $\left(a_{1}, \ldots, a_{k}\right)$ such that $\sum_{l=1}^{k} a_{l}=v(N)$. Consider the following TU games: $\left(N, w^{1}\right),\left(N, w^{2}\right), \ldots,\left(N, w^{k}\right)$, where

$$
\begin{aligned}
& w^{1}(S):=\left\{\begin{array}{cl}
a_{1} & \text { if } S=N \\
\min \left\{a_{1}, v(S)\right\} & \text { otherwise }
\end{array}\right. \\
& w^{2}(S):=\left\{\begin{array}{cc}
a_{2} & \text { if } S=N \\
\min \left\{a_{2}, \max \left[0, v(S)-\sum_{i \in S} \phi_{i}\left(N, w^{1}\right)\right]\right\} & \text { otherwise }
\end{array}\right. \\
& w^{l}(S):=\left\{\begin{array}{cc}
a_{l} & \text { if } S=N \\
\min \left\{a_{l}, \max \left[0, v(S)-\sum_{m=1}^{l-1} \sum_{i \in S} \phi_{i}\left(N, w^{m}\right)\right]\right\} & \text { otherwise }
\end{array}\right.
\end{aligned}
$$

Then $\phi(N, v)=\sum_{m=1}^{k} \phi\left(N, w^{m}\right)$.

Sketch of proof. In the lemma, we take $v(N)$ and divide it in $k$ positive parts, where $k$ is a finite number. We then compute the serial rule for each of the $k$ games, and see that each player gets the same in total as in the serial rule of the original game. The $k$ games are formed as follows: $w^{l}(N)$ is always $a_{l}$ for each $l: 1, \ldots, k$; the other coalitions have $v(S)$ minus what has been distributed so far according to the serial rule of the previous games, unless this would be negative (in which case the value is 0 ) or above $w^{l}(N)$ (in which case the value is $a_{l}$ ). Denote by $d_{i}^{l}$ the $d$-value for player $i$ associated to the auxiliary game $\left(N, w^{l}\right)$. 
The idea of the proof is that player $i$ cannot get anything until $d_{i}$ is distributed and becomes a veto player from then on. For example, if $a_{1} \leq d_{i}$, any coalition $S$ associated to $d_{i}$ has $w^{1}(S)=\min \left(v(S), a_{1}\right)=\min \left(d_{i}, a_{1}\right)=$ $a_{1}$. Hence, $d_{i}^{1}=a_{1}=w^{1}(N)$ and player $i$ gets nothing according to the serial rule of $w^{1}$. The entire payoff $a_{1}$ will go to players with $d_{j}^{1}<a_{1}$. Such players must have $d_{j}<a_{1} \leq d_{i}$ and must belong to any $T$ such that $v(T)>a_{1}$ (otherwise $d_{j}^{1} \geq a_{1}$ ). Hence, any coalition with positive value in $w^{2}$ will have $w^{2}(T)=\min \left(v(T)-a_{1}, a_{2}\right)$ and any player who got a positive payoff according to $\phi\left(N, w^{1}\right)$ will be a veto player from $w^{2}$ onwards.

The same holds for $w^{2}$ if $a_{1}+a_{2} \leq d_{i}$. Any coalition $S$ with $v(S)=d_{i}$ has $w^{2}(S)=\min \left(v(S)-a_{1}, a_{2}\right)=a_{2}$, hence $d_{i}^{2}=a_{2}=w^{2}(N)$ and player $i$ gets nothing according to $\phi\left(N, w^{2}\right)$. The entire $a_{2}$ goes to players with lower $d_{j}^{2}$-values, and these players must have $d_{j}<a_{1}+a_{2} \leq d_{i}$ and must belong to any coalition $T$ such that $v(T)-a_{1}>a_{2}$. Hence, any coalition with positive value in $w^{3}$ will have $w^{3}(T)=\min \left(v(T)-a_{1}-a_{2}, a_{3}\right)$ and any player who got a positive payoff in $\phi\left(N, w^{2}\right)$ will be a veto player from $w^{3}$ onwards.

Let $l$ be the first auxiliary game for which $a_{1}+\ldots+a_{l}>d_{i}$. At this point, the value of any coalition $S$ associated to $d_{i}$ is $w^{l}(S)=\min (v(S)-$ $\left.\sum_{t=1}^{l-1} a_{t}, a_{l}\right)=v(S)-\sum_{t=1}^{l-1} a_{t}<a_{l}=w^{l}(N)$. The value $d_{i}^{l}=w^{l}(S)$ will still be divided between the other players, but above that value player $i$ will receive a share. Note that $\sum_{t=1}^{l} d_{i}^{t}=d_{i}$. Hence, player $i$ finds that $d_{i}$ is divided (not necessarily equally) between players with a lower index. Any player with $\phi_{j}\left(N, w^{l}\right)>0$ must have $d_{j}^{l}<a_{l}$, and thus must belong to any coalition $T$ such that $v(T)-\sum_{t=1}^{l-1} a_{t}>a_{l}$. Hence, any $T$ with $w^{l+1}(T)>0$ will have $w^{l+1}(T)=\min \left(v(T)-\sum_{t=1}^{l} a_{t}, a_{l+1}\right)$ and any $j$ with $\phi_{j}\left(N, w^{l}\right)>0$ -including player $i$ - will be a veto player from $w^{l+1}$ onwards and will therefore receive a share of the payoff.

We see that players receive a positive share if and only if $d_{i}$ has already been distributed. Above $d_{i}$, the serial rules of the auxiliary games ensure that the payoff is evenly divided between the players who are getting a positive share. This process replicates the serial rule of the original game. For the same reason, if $\sum_{l=1}^{k} a_{l}<v(N)$, the proposer will get less than $\phi_{1}(N, v)$.

Note that lemma 10 refers to a sequence of TU games such that each game 
is obtained after distributing the serial rule payoffs for the previous game; the games $w^{t}$ in lemma 9 are obtained by subtracting balanced proposals from $w^{t-1}$. It turns out that the TU games involved are identical in both cases: the sequence $w^{t}$ depends only on the total amounts distributed $x^{1}(N), \ldots, x^{T}(N)$ (denoted by $a_{1}, \ldots, a_{k}$ in lemma 10). This is because the set of players that get a positive payoff at period $t$ is the same in both cases (lemma 7) and all these players become veto players at period $t+1$ (lemma 6 ). Hence, any coalition with positive value at $t$ has $w^{t}(S)=\min \left(w^{t-1}(S)-x^{t-1}(N), x^{t}(N)\right)$ in both cases.

Putting the above lemmas together we can prove theorem 2. First, any payoff player 1 can achieve in a MBRE can be achieved by balanced proposals (lemma 4). Second, given that proposals are balanced, the payoff player 1 can get cannot exceed the sum of the serial rules of the games $w^{t}$ (lemma 9). Since the sum of the serial rules of the games $w^{t}$ cannot exceed the serial rule of the original game (lemma 10), player 1 can never get more than $\phi_{1}(N, v)$ in a MBRE. Also, player 1 can only get $\phi_{1}(N, v)$ if all other players get their serial rule payoff (corollary 3 ). Finally, $\phi(N, v)$ is achievable by the sequential proposals described in lemma 5 .

Note that the assumption $T \geq n$ only plays a role in lemma 5 . For time horizons shorter than $n-1$, all auxiliary results still hold but player 1 may not be able to achieve a payoff as high as $\phi_{1}(N, v)$.

\subsection{Proof of theorem 3}

We start by pointing out some immediate consequences of the results in section 3.3 .

Corollary 4 Let $(N, v)$ be a veto balanced $T U$ game and $G^{T}(N, v)$ its associated extensive form game with $T \geq n$. Let $z=\sum_{t=1}^{T} x^{t}$ be an outcome resulting from some SPE of the game $G^{T}(N, v)$. If $z$ differs from $\phi(N, v)$ then $z_{1}$ cannot be achieved by making balanced proposals.

Suppose to the contrary that there is an SPE outcome $z$ that differs from $\phi(N, v)$ and can be achieved by balanced proposals. If $z$ differs from $\phi(N, v)$, 
$z_{1} \geq \phi_{1}(N, v)$ (otherwise the proposer would prefer to play the strategy described in lemma 5 , which is available since $T \geq n$ ). If $z$ is achievable by balanced proposals, it is (trivially) achievable under myopic responder behavior. However, under myopic behavior of the responders, the proposer can only achieve at least $\phi_{1}(N, v)$ if all players are getting their serial rule payoffs, that is, if $z=\phi(N, v)$ (theorem 2), a contradiction.

Corollary 5 Let $(N, v)$ be a veto balanced $T U$ game and $G^{T}(N, v)$ its associated extensive form game with $T \geq n$. Let $z=\sum_{t=1}^{T} x^{t}$ be an outcome resulting from some SPE of the game $G^{T}(N, v)$. If $z$ differs from $\phi(N, v)$ then there exists at least one period $t$ and one player $p$ for which $f_{1 p}\left(x^{t},\left(N, v^{t}\right)\right)>x_{p}^{t} \geq 0$.

This is because if $x_{l}^{t} \geq f_{1 l}\left(x^{t}, v^{t}\right)$ for all $l$ and $t, z$ would be achievable under myopic behavior of the responders by proposing $x^{t}$ in each period $t$ (lemma 3), but we know from theorem 2 that the proposer can only get at least $\phi_{1}(N, v)$ if all players are getting their serial rule payoffs.

Since myopic behavior always leads to $f_{1 i}\left(x^{t}, v^{t}\right) \leq x_{i}^{t}$ for all $i$ and all $t$ (lemma 3), the presence of a player $p$ for which $f_{1 p}\left(x^{t},\left(N, v^{t}\right)\right)>x_{p}^{t}$ indicates non-myopic responder behavior. This responder may be player $p$ (nonmyopically accepting a proposal), or a responder moving after $p$ (nonmyopically rejecting a proposal and transferring payoff to player 1). In example 3, player 3 rejects a proposal nonmyopically and as a result creates the inequality $f_{12}\left(x^{t}, v^{t}\right)>x_{2}^{t}$ at the end of period $t=4$.

We are now ready to prove theorem 3 . We will show in lemma 14 that, if responders move following the order of nonincreasing $d$-values of $v^{t}$, any SPE outcome $z$ is such that the proposer can obtain $z_{1}$ by making balanced proposals. Since any SPE outcome $z$ different from $\phi(N, v)$ would be unachievable with balanced proposals according to corollary 4 , this will complete the proof.

In order to prove lemma 14 we need several auxiliary results.

We denote by $x^{t, i}$ the proposal that emerges in period $t$ immediately after $i$ gets the move. The following lemma establishes a property of $x^{t, i}$ that must be inherited by the final outcome in period $t, x^{t}$. 
Lemma 11 Suppose after player $i$ responds to the proposal in period $t$ it holds that $f_{1 i}\left(x^{t, i}, v^{t}\right)>0$. Then $f_{1 i}\left(x^{t}, v^{t}\right)>0$ regardless of the responses of the players moving after $i$.

Proof. Suppose by contradiction that $f_{1 i}\left(x^{t}, v^{t}\right) \leq 0$. This means that at the end of period $t$ there is a coalition $S^{*}$ such that $i \notin S^{*}$ and $v^{t}\left(S^{*}\right) \geq$ $x^{t}\left(S^{*}\right)$. Because $f_{1 i}\left(x^{t, i}, v^{t}\right)>0$ immediately after $i$ responds to the proposal, all coalitions excluding $i$ had a positive satisfaction at that point, and in particular $v^{t}\left(S^{*}\right)<x^{t, i}\left(S^{*}\right)$. There must be a player $h$ moving after $i$ such that $h \notin S^{*}$ and $h$ has received a payoff transfer from player 1 by rejecting the proposal. At the moment of rejection by $h$ we have $f_{1 h}\left(x^{t, h}, v^{t}\right)=x_{h}^{t}>0$. However, since $S^{*}$ can be used by 1 to complain against $h$, at the end of period $t$ we have $f_{1 h}\left(x^{t}, v^{t}\right) \leq 0$. There must be another player $l$ moving after $h$ that has received a payoff transfer from player 1, and this player cannot be in $S^{*}$. Then this player is in the same situation as player $h$ : he has $f_{1 l}\left(x^{t, l}, v^{t}\right)>0$ at the moment of rejection, but at the end of period $t$ he has $f_{1 l}\left(x^{t}, v^{t}\right) \leq 0$. Thus there must be another player moving after him that has caused this change and would himself be in the same situation as player $h \ldots$ but the number of players is finite.

Note that lemma 11 holds for any strategy profile, not necessarily an equilibrium.

The next auxiliary result will allow us to compare the equilibria of games with different characteristic functions. If one of the characteristic functions is "worse" than the other (in the sense of having lower values), player 1 might still have a greater SPE payoff, but only with nonmyopic responder behavior.

Lemma 12 Let $(N, v)$ and $(N, w)$ be two veto balanced games in which player 1 is a veto player. Let $w(S) \geq v(S)$ for any $S$. Let $G^{T}(N, v)$ and $G^{T}(N, w)$ be the associated extensive form games with $T$ proposals. If the payoff provided to the proposer by an SPE outcome of the game $G^{T}(N, w)$ is strictly lower than the payoff provided to the proposer by an SPE outcome of the game $G^{T}(N, v)$, then the SPE outcome of $G^{T}(N, v)$ is such that $x_{l}^{t}<f_{1 l}\left(x^{t}, v^{t}\right)$ for some $l$ and $t$, which implies that at least one responder is behaving nonmyopically. 
Proof. By contradiction, suppose the final payoffs in the SPE outcome of $G^{T}(N, v)$ are such that $x_{l}^{t} \geq f_{1 l}\left(x^{t}, v^{t}\right)$ for all $l$ and $t$.

Consider the strategy combination (not necessarily an equilibrium) of the game $G^{T}(N, w)$ in which the proposer makes the sequence of proposals $x^{t}$ and responders accept. Because by assumption $w(S) \geq v(S)$ for all $S$, $w^{t}(S) \geq v^{t}(S)$ for all $t$ given this strategy combination and thus $f_{1 l}\left(x^{t}, w^{t}\right) \leq$ $f_{1 l}\left(x^{t}, v^{t}\right) \leq x_{l}^{t}$ for all $l$ and $t$.

Given that $f_{1 l}\left(x^{t}, w^{t}\right) \leq x_{l}^{t}$ for all $l$ and $t$ in this strategy profile, we can use lemma 4 to construct a sequence of balanced proposals $y^{t}$ for the game $G^{T}(N, w)$ where the payoff of the proposer does not change, that is, $\sum_{t=1}^{T} y_{1}^{t}=\sum_{t=1}^{T} x_{1}^{t}$. Because the proposer always has the option of making the sequence of balanced proposals $y^{t}$ in the game $G^{T}(N, w)$, the proposer's payoff in any SPE of game $G^{T}(N, w)$ must be at least $\sum_{t=1}^{T} y_{1}^{t}=\sum_{t=1}^{T} x_{1}^{t}$.

We have shown that if an SPE outcome of $G^{T}(N, v)$ is such that $x_{l}^{t} \geq$ $f_{1 l}\left(x^{t}, v^{t}\right)$ for all $l$ and $t$, then the payoff obtained by the proposer in this outcome can also be obtained with balanced proposals (and therefore in any SPE) in $G^{T}(N, w)$. Hence, if the proposer is obtaining strictly less in an SPE of $G^{T}(N, w)$, it must be the case that $x_{l}^{t}<f_{1 l}\left(x^{t}, v^{t}\right)$ for some $l$ and $t$.

The next auxiliary result provides a bound for the payoff difference between player 1 and player $i \neq 1$.

Lemma 13 Let $(N, v)$ be a veto balanced TU game. Consider the associated game with $T$ periods $G^{T}(N, v)$. Fix a period $l \in\{1, \ldots, T\}$ and a subgame that starts in period l (not necessarily on the equilibrium path), and label the responders according to the nondecreasing order of d-values in the game $v^{l}$. Let $y^{l}=\sum_{t=l}^{T} x^{t}$ be the vector of payoffs accumulated between $l$ and T. Then $y_{i}^{l} \geq y_{1}^{l}-d_{i}^{l}$ for all $i \in\{2, \ldots, n\}$ in any SPE of $G^{T}(N, v)$. Moreover, the inequality is strict if $d_{i}^{l}>d_{2}^{l}$.

Proof. Note that, because period $T$ is the last period of the game, myopic and rational behavior coincide, so $x_{i}^{T} \geq f_{1 i}\left(v^{T}, x^{T}\right)$ for all $i$. 
Consider player 2. Since players play myopically in period $T$, it must be the case that ${ }^{13}$

$$
\begin{gathered}
x_{2}^{T} \geq f_{12}\left(x^{T}\right) \geq x_{1}^{T}-\left(d_{2}^{l}-\sum_{t=l}^{T-1} x_{1}^{t}\right)= \\
=\sum_{t=l}^{T} x_{1}^{t}-d_{2}^{l}=y_{1}^{l}-d_{2}^{l} .
\end{gathered}
$$

Since $y_{2}^{l} \geq x_{2}^{T}$, it follows that $y_{2}^{l} \geq y_{1}^{l}-d_{2}^{l}$.

Now consider player $i \neq 2$. There are two possible cases, depending on whether $y_{1}^{l} \leq d_{2}^{l}$.

If $y_{1}^{l} \leq d_{2}^{l}$, the result follows immediately since

$$
y_{1}^{l}-d_{i}^{l} \leq y_{1}^{l}-d_{2}^{l} \leq 0 \leq y_{i}^{l} .
$$

It is also clear that the inequality is strict if $d_{2}^{l}<d_{i}^{l}$.

From now on we assume $y_{1}^{l}>d_{2}^{l}$. Note that since we have already shown that $y_{2}^{l} \geq y_{1}^{l}-d_{2}^{l}$, it follows that that $y_{2}^{l}>0$ in this case. There are again two possible cases, depending on whether the coalition associated to $f_{1 i}\left(x^{T}, v^{T}\right)$ contains 2 .

If the coalition contains 2 , we have

$$
\begin{aligned}
y_{i}^{l} & \geq x_{i}^{T} \geq f_{1 i}\left(x^{T}, v^{T}\right) \geq x_{1}^{T}+x_{2}^{T}-\left(d_{i}^{l}-\sum_{t=l}^{T-1} x_{1}^{t}-\sum_{t=l}^{T-1} x_{2}^{t}\right)= \\
& =y_{1}^{l}+y_{2}^{l}-d_{i}^{l}>y_{1}^{l}-d_{i}^{l},
\end{aligned}
$$

where the last inequality follows from the fact that $y_{2}^{l}>0$.

If the coalition does not contain 2 , we have $f_{1 i}\left(x^{T}\right) \geq f_{12}\left(x^{T}\right)$. Then

$$
y_{i}^{l} \geq x_{i}^{T} \geq f_{1 i}\left(x^{T}, v^{T}\right) \geq f_{12}\left(x^{T}, v^{T}\right) \geq y_{1}^{l}-d_{2}^{l} \geq y_{1}^{l}-d_{i}^{l} .
$$

Note that the inequality is strict for $d_{i}^{l}>d_{2}^{l}$.

The main building block of the proof is the next lemma, which shows that, given the particular order of responders we impose, the proposer cannot do better than with balanced proposals.

\footnotetext{
${ }^{13}$ If $S$ is a coalition associated to $f_{12}\left(x^{T}\right)$, the total payoff of $S$ must be at least $x_{1}^{T}$. Also, the total value of $S$ must be at most $d_{2}^{l}-\sum_{t=l}^{T-1} x_{1}^{t}$.
} 
Lemma 14 Let $(N, v)$ be a veto balanced TU game. Consider the associated game $G^{T}(N, v)$ in which the responders move following the order of nonincreasing $d$-values of $v^{t}$. Let $z=\sum_{t=1}^{T} x^{t}$ be an outcome resulting from some SPE of the game $G^{T}(N, v)$. Then the proposer can obtain $z_{1}$ by making balanced proposals.

Proof. Suppose on the contrary that $z_{1}$ cannot be obtained with balanced proposals. By Lemma 4 we know that there is a player $k$ and a stage $t$ such that $f_{1 k}\left(x^{t}, v^{t}\right)>x_{k}^{t} \geq 0$; otherwise the proposer can obtain $z_{1}$ with balanced proposals.

Let $t$ be the last period ${ }^{14}$ in which for some responder it holds that $f_{1 k}\left(x^{t}, v^{t}\right)>x_{k}^{t} \geq 0$. Let $k$ be the last responder at $t$ for whom $f_{1 k}\left(x^{t}, v^{t}\right)>$ $x_{k}^{t} \geq 0$. We consider two cases:

Case a) There is a player $p$ with $d_{p}^{t} \leq d_{k}^{t}$ such that $f_{1 p}\left(x^{t}, v^{t}\right) \leq 0$. Note that $f_{1 k}\left(x^{t}, v^{t}\right)>x_{k}^{t} \geq 0$ means that any coalition without player $k$ has a positive satisfaction and, in particular any coalition $S_{k} \in \arg \max _{S \subseteq N \backslash\{k\}} v^{t}(S)$. On the other hand since $f_{1 p}\left(x^{t}, v^{t}\right) \leq 0$ then there exists a coalition without player $p$ for which the satisfaction is not positive. Let $S_{p}^{*}$ be one such coalition (it must contain $k$ ). Then we have the following two inequalities:

$$
x^{t}\left(S_{k}\right)>d_{k}^{t} \text { and } v^{t}\left(S_{p}^{*}\right) \geq x^{t}\left(S_{p}^{*}\right) .
$$

Combining the two inequalities we obtain

$$
x^{t}\left(S_{k}\right)-x^{t}\left(S_{p}^{*}\right)>d_{k}^{t}-v^{t}\left(S_{p}^{*}\right) \geq d_{k}^{t}-d_{p}^{t} \geq 0 .
$$

The inequality above implies that there are players not in $S_{p}^{*}$ receiving a positive payoff in period $t$.

Consider a new allocation, $y^{t}$, which is identical to $x^{t}$ except that $y_{i}^{t}=$ 0 for all $i$ not in $S_{p}^{*}$ (thus $y_{i}^{t}=x_{i}^{t}$ for all $i$ in $S_{p}^{*}$ ). We now show that $f_{1 i}\left(y^{t}, v^{t}\right) \leq y_{i}^{t}$ for all $i \in N$, so that player 1 can get the same payoff with balanced proposals by lemma 4 .

\footnotetext{
${ }^{14}$ It is clear that $t<m$, since all responders behave as myopic maximizers in the last period.
} 
For any player $i$ it holds that $f_{1 i}\left(y^{t}, v^{t}\right) \leq f_{1 i}\left(x^{t}, v^{t}\right)$.

Because $S_{p}^{*}$ can be used against any player outside $S_{p}^{*}$, for any player outside $S_{p}^{*}$ it holds that $f_{1 i}\left(y^{t}, v^{t}\right) \leq f_{1 p}\left(y^{t}, v^{t}\right) \leq f_{1 p}\left(x^{t}, v^{t}\right) \leq 0$. Thus, $f_{1 i}\left(y^{t}, v^{t}\right) \leq y_{i}^{t}$ for all $i \notin S_{p}^{*}$.

We can also rule out the existence of a player $l \in S_{p}^{*}$ for which $f_{1 l}\left(y^{t}, v^{t}\right)>$ $y_{l}^{t}$. If there was such a player, this inequality would have already existed for $x^{t}$, since $f_{1 l}\left(y^{t}, v^{t}\right) \leq f_{1 l}\left(x^{t}, v^{t}\right)$ and $y_{l}^{t}=x_{l}^{t}$. Since player $k$ is the last player satisfying the inequality for $x^{t}$, it must be the case that $d_{l}^{t} \geq d_{k}^{t} \geq d_{p}^{t}$, thus we can repeat the reasoning above with $S_{l}$ and $S_{p}^{*}$ and, given that nothing has changed for $S_{p}^{*}$, we would conclude that $y^{t}\left(S_{l}\right)-y^{t}\left(S_{p}^{*}\right)>0$, a contradiction since all players outside $S_{p}^{*}$ have zero payoffs. Thus, $f_{1 i}\left(y^{t}, v^{t}\right) \leq y_{i}^{t}$ for all $i$.

Note that for this part of the proof no assumption is needed about the order in which the responders move. ${ }^{15}$

Case b) The second case is $f_{1 l}\left(x^{t}, v^{t}\right)>0$ for all players moving after $k$ at $t$. By assumption, on the equilibrium path from $t+1$ onwards all proposals have an associated balanced proposal. We distinguish two subcases:

b1) The last player to act nonmyopically at $t$ has accepted a proposal. This player must be player $k$ or a player moving after $k$. Call this player $p$ ( $p$ moving after $k$ is possible if a myopic rejection by a player moving after $p$ has restored $f_{1 p} \leq x_{p}$ ).

We will show that it is not in $p$ 's interest to accept the proposal. To do this, we need to analyze two subgames: the subgame on the equilibrium path in which $p$ accepts the proposal, and the subgame off the equilibrium path in which $p$ rejects the proposal. We will talk about the $A$-path (the equilibrium path) and the $R$-path. Denote by $x^{A, t}$ and $x^{R, t}$ the final payoffs in period $t$ depending on whether player $p$ accepts or rejects the proposal. If player $p$ rejects the proposal, we take any subgame perfect equilibrium of that subgame. Denote by $v^{A, t+1}$ and $v^{R, t+1}$ the corresponding TU games at $t+1$.

Because $f_{1 l}\left(x^{t}, v^{t}\right)>0$ for $l \in\{2, \ldots, p\}$, on the $A$-path all players in $\{2, \ldots, p\}$ are veto players at $t+1$.

\footnotetext{
${ }^{15}$ All we need to assume in this part of the proof is that player $k$ is the "last" player in the sense of being the player with the lowest $d_{i}^{t}$, not necessarily the one who moves last.
} 
The game $v^{R, t+1}$ is better than the game $v^{A, t+1}$ (in the sense of lemma 12). If $v^{A, t+1}(S)>0$, coalition $S$ must contain all players in $\{1,2, \ldots, p\}$. For this kind of coalition $v^{R, t+1}(S)=v^{A, t+1}(S)$, since any payoff transfers after rejection occur between members of $\{1, \ldots, p\}$ (here the order of moves is essential). Thus, $v^{A, t+1}(S) \leq v^{R, t+1}(S)$ for all $S$.

We now show that $p$ is also veto at $t+1$ on the $R$-path.

Suppose $p$ is not veto at $t+1$ on the $R$-path. Then there is a coalition $S_{p}$ such that $p \notin S_{p}$ and $v^{R, t+1}\left(S_{p}\right)>0$. This can only happen if $v^{R, t}\left(S_{p}\right)>$ $x^{R, t}\left(S_{p}\right)$, or equivalently $f_{1 p}\left(x^{R, t}, v^{t}\right)<0$, contradicting lemma 11 .

Thus, player $p$ is a veto player at $t+1$ regardless of whether he accepts or rejects the proposal. Given the order of moves, veto players can secure at least the same payoff as the proposer. There is no reason for veto players to act nonmyopically because the game at $t+2$ will be the same regardless of how the payoff is distributed at $t+1$ between veto players; no payoff can go to anyone else given the order of responders. For the same reason the proposer will never make a proposal that gives another veto player more than he gets himself, so that all veto players must get the same payoff given the order of moves. Let $y_{1}^{R}$ be player 1's payoff if $p$ rejects the proposal (this is the payoff accumulated between periods $t+1$ and $n$ ) and $y_{1}^{A}$ be player 1 's payoff if $p$ accepts the proposal. Because of lemma 12, the only way in which $y_{1}^{A}$ can exceed $y_{1}^{R}$ is if there is a nonmyopic move at $v^{A, t+1}$ that leads to $f_{1 i}\left(x^{A, t+1}, v^{A, t+1}\right)>x_{i}^{A, t+1}$ for some $i$. By assumption this is not the case. Thus, it was not in $p$ 's interest to accept: rejecting would yield a higher payoff at $t$, and at least the same payoff in the rest of the game.

b2) The last player to act nonmyopically at $t$ has rejected a proposal. Let $p$ be the last player to act nonmyopically at $t$. This player cannot be player $k$ because after any rejection (myopic or otherwise) it holds that $f_{1 k}($. $x_{k}^{t}$, and given that the remaining responders act myopically this inequality would never be reversed. Someone moving after $k$ must have rejected a proposal nonmyopically (transferring payoff to the proposer) and created the inequality $f_{1 k}\left(x^{t}, v^{t}\right)>x_{k}^{t}$, hence player $p$ must be moving after $k$. We will show that it is not in $p$ 's interest to reject the proposal. To do this, we need to analyze two subgames: the subgame on the equilibrium path in which $p$ 
rejects the proposal, and the subgame off the equilibrium path in which $p$ accepts the proposal. We will talk about the $R$-path (the equilibrium path) and the $A$-path.

Because $f_{1 l}\left(x^{t}, v^{t}\right)>0$ for $l \in\{2, \ldots, k\}$, on the equilibrium path all players in $\{2, \ldots, k\}$ are veto players at $t+1$.

The game $v^{A, t+1}$ is better than the game $v^{R, t+1}$ (in the sense of lemma 12). If $v^{R, t+1}(S)>0$, coalition $S$ must contain all players in $\{1,2, \ldots, p\}$. For this kind of coalition $v^{R, t+1}(S)=v^{A, t+1}(S)$, since any payoff transfers on the $A$-path must occur between members of $\{1,2, \ldots, p\}$ (again, here the order of moves is essential). Thus, $v^{R, t+1}(S) \leq v^{A, t+1}(S)$ for all $S$.

Suppose player $p$ is veto also on the $A$-path. Then the reasoning of case b1 applies, and there is no reason for $p$ to act nonmyopically in period $t$.

Now suppose player $p$ is not veto on the $A$-path at $t+1$. We define $d_{p}^{A, t+1}:=\max _{S \subseteq N \backslash\{p\}} v^{A, t+1}(S)$. Since $p$ is not a veto player at $t+1$ on the $A$-path, $d_{p}^{A, t+1}>0$. There is a coalition $S_{p}$ such that $v^{t}\left(S_{p}\right)-x^{A, t}\left(S_{p}\right)=d_{p}^{A, t+1}>0$. Since by assumption $f_{1 p}\left(x^{t}, v^{t}\right)>0$ on the $R$-path, we also have $x^{R, t}\left(S_{p}\right)-$ $v^{t}\left(S_{p}\right)>0$. From the two inequalities we get $x^{R, t}\left(S_{p}\right)-x^{A, t}\left(S_{p}\right)>d_{p}^{A, t+1}$.

Let $\alpha$ be the payoff player $p$ transfers to player 1 when rejecting the proposal (part of this payoff may then go to other players between 2 and $p-1$ if they myopically reject a proposal). We want to show that $\alpha \geq$ $x^{R, t}\left(S_{p}\right)-x^{A, t}\left(S_{p}\right)$, which implies $\alpha>d_{p}^{A, t+1}$. This is not completely obvious because part of the difference could be due to a player outside $S_{p}$ myopically rejecting on the $A$-path.

Claim. $\alpha \geq x^{R, t}\left(S_{p}\right)-x^{A, t}\left(S_{p}\right)>d_{p}^{A, t+1}$.

Note that $x^{R, t}$ and $x^{A, t}$ are identical for all responders moving before $p$. Because of the order of moves, any payoff transfers due to a change of $p$ 's action from $\mathrm{R}$ to $\mathrm{A}$ occur within the set $\{1,2, \ldots, p\}$. Denote by $T$ the set $\{2, \ldots, p-1\} \backslash S_{p}$. We can write $x^{R, t}\left(S_{p}\right)+x^{R, t}(T)=x^{A, t}\left(S_{p}\right)+x^{A, t}(T)+\alpha$. All we need to show is that $x_{h}^{R, t} \geq x_{h}^{A, t}$ for all $h \in T$ (this is obvious if $T$ is empty). This implies $x^{R, t}(T) \geq x^{A, t}(T)$ and hence $x^{R, t}\left(S_{p}\right)-x^{A, t}\left(S_{p}\right) \leq \alpha$.

Suppose there is a player $h$ in $T$ that has $x_{h}^{R, t}<x_{h}^{A, t}$. Since this player is not in $S_{p}, S_{p}$ can be used by player 1 against him. 
Player $h$ must have rejected in the $A$-path (if he had accepted he would have $\left.x_{h}^{R, t} \geq x_{h}^{A, t}\right)^{16}$. After rejecting he is left with $f_{1 h}()=.x_{h}^{A, t}>0$. On the other hand, $v^{t}\left(S_{p}\right)-x^{A, t}\left(S_{p}\right)>0$. Thus, $f_{1 h}\left(x^{A, t}, v^{t}\right)<0$, contradicting lemma 11.

Now we are in a position to compare payoffs on the $A$ and $R$-paths and see that $p$ prefers to accept the proposal.

Since player $p$ is veto on the $R$-path, he gets $y_{1}^{R}$. On the $A$-path, he gets $y_{p}^{A}$, whereas the proposer gets $y_{1}^{A}$. Because lemma 13 applies to all subgames regardless of whether they are on the equilibrium path, $y_{p}^{A} \geq y_{1}^{A}-d_{p}^{A, t+1}$.

In order to have an equilibrium, $p$ must prefer to reject the proposal, thus we need $x_{p}^{t}+y_{1}^{R} \geq x_{p}^{t}+\alpha+y_{p}^{A}>x_{p}^{t}+d_{p}^{A, t+1}+y_{1}^{A}-d_{p}^{A, t+1}$. Therefore we need $y_{1}^{R}>y_{1}^{A}$. Since the game $v^{A, t+1}$ is better than the game $v^{R, t+1}$ (in the sense of lemma 12), and by assumption $f_{1 i}\left(x^{l}, v^{l}\right)>x_{i}^{l}$ never happens on the $R$-path from $t+1$ onwards, the inequality $y_{1}^{R}>y_{1}^{A}$ cannot hold.

We have shown that any SPE outcome is such that the proposer can always achieve $z_{1}$ with balanced proposals. From the previous section we know that the best outcome the proposer can achieve with balanced proposals is $\phi(N, v)$, hence this completes the proof.

\section{Acknowledgements}

We thank Jeroen Kuipers, Juan J. Vidal Puga, Alex Possajennikov and Elena Inarra for their helpful comments. Javier Arin acknowledges financial support from the Spanish Government (project ECO2012-31346) and from the Basque Government (project IT568-13). This author also is also grateful for the hospitality provided by the Department of Economics of the University of Rochester and the financial support provided by the Program "Salvador de Madariaga" of the Ministry of Education and Science of Spain. We also thank two anonymous referees for helpful comments and suggestions.

\footnotetext{
${ }^{16} \mathrm{By}$ assumption, $p$ is the last player to behave nonmyopically on the R-path. This leaves us with two possibilities for $h$ on the R-path: myopic acceptance and myopic rejection. In neither case it is possible for $h$ to get a higher payoff by accepting the proposal on the A-path.
} 


\section{References}

[1] Arin J. and Feltkamp V. (1997) The nucleolus and kernel of veto-rich transferable utility games. International Journal of Game Theory 26: 61-73.

[2] Arin J. and Feltkamp V. (2007) Coalitional games with veto players: consistency, monotonicity and Nash outcomes. Journal of Mathematical Economics 43: 855-870.

[3] Arin J. and Feltkamp V. (2012) Coalitional games: monotonicity and core. European Journal of Operational Research 216: 208-213.

[4] Arin J. and Katsev I. (2014) The SD-Prenucleolus for TU games. Mathematical Methods of Operations Research 80: 307-327.

[5] Chang C. and Hu C.-C. (2008) A non-cooperative interpretation of the f-just rules of bankruptcy problems. Games and Economic Behavior 63: 133-144.

[6] Chun Y. (1989) A noncooperative justification for egalitarian surplus sharing. Mathematical Social Sciences 17: 245-261.

[7] Dagan N., Serrano R. and Volij O. (1997) A non-cooperative view of consistent bankruptcy rules. Games and Economic Behavior 18: 55-72.

[8] Davis M. and Maschler M. (1965) The kernel of a cooperative game. Naval Research Logistics Quarterly 12: 223-259.

[9] Driessen T., Muto S. and Nakayama M. (1992) A cooperative game of information trading: The core, the nucleolus and the kernel. Zeitschrift für Operations Research 36: 55-72.

[10] Gilles R., Owen G. and van den Brink R. (1992) Games with permission structures: the conjunctive approach. International Journal of Game Theory 20: 277-293. 
[11] Herrero C., Moreno-Ternero J.D. and Ponti G. (2010) On the adjudication of conflicting claims: an experimental study. Social Choice and Welfare 34: 145-179.

[12] Karagözoğlu, E. (2014) A noncooperative approach to bankruptcy problems with an endogenous estate. Annals of Operations Research 217: 299-318.

[13] Maschler M. (1992) The bargaining set, kernel and nucleolus. Handbook of game theory with economic applications I, Aumann R. J. and Hart S. eds., Amsterdam: North-Holland.

[14] Meggido N. (1974) On the nonmonotonicity of the bargaining set, the kernel and the nucleolus of a game. SIAM Journal of Applied Mathematics 27: 355-358.

[15] Moulin H. and Shenker S. (1992) Serial cost sharing. Econometrica 60: 1009-37.

[16] Muto S. (1986) An information good market with symmetric externalities. Econometrica 54: 295-312.

[17] Muto S., Potters J. and Tijs S. (1989) Information market games. International Journal of Game Theory 18: 209-226.

[18] O'Neill, B. (1982) A problem of rights arbitration from the Talmud. Mathematical Social Sciences 2: 345-371.

[19] Peleg B. (1986) On the reduced game property and its converse. International Journal of Game Theory 15: 187-200.

[20] Peleg B. and Sudhölter P. (2003) Introduction to the theory of cooperative games. Kluwer Academic Publishers.

[21] Schmeidler D. (1969) The nucleolus of a characteristic function game. SIAM Journal of Applied Mathematics 17: 1163-117. 
[22] Serrano R. (1995) Strategic bargaining, surplus sharing problems and the nucleolus. Journal of Mathematical Economics 24: 319-329.

[23] Shapley L. S. and Shubik M. (1967) Ownership and the production function. Quarterly Journal of Economics 81: 88-111. 